\begin{tabular}{|c|c|}
\hline Title & $\begin{array}{l}\text { A ngular distributions of absorbed dose of Bremsstrahlung and secondary electrons induced by } 18,28 \text { and } 38 \mathrm{MeV} \\
\text { electron beams in thick targets }\end{array}$ \\
\hline Author(s) & $\begin{array}{l}\text { Takada, Masashi; Kosako, Kazuaki; Oishi, Koji; Nakamura, Takashi; Sato, Kouichi; Kamiyama, Takashi; Kiyanagi, } \\
\text { Y oshiaki }\end{array}$ \\
\hline Citation & $\begin{array}{l}\text { Radiation Protection Dosimetry, 153(3), 369-383 } \\
\text { https://doi.org/10.1093/ppd/hcs114 }\end{array}$ \\
\hline Issue Date & $2013-03$ \\
\hline Doc URL & http:/hdl.handle.net/2115/54776 \\
\hline Rights & $\begin{array}{l}\text { This is a pre copy-editing, author-produced PDF of an article accepted for publication in Radiation Protection } \\
\text { Dosimetry following peer review. The definitive publisher-authenticated version A ngular distributions of absorbed dose } \\
\text { of Bremsstrahlung and secondary electrons induced by } 18,28 \text { and } 38-\mathrm{MeV} \text { electron beams in thick targets is available } \\
\text { online at: http://ppd.oxfordjournal s.org/content/153/3/369.full. }\end{array}$ \\
\hline Type & article (author version) \\
\hline File Information & mtakada.pdf \\
\hline
\end{tabular}

Instructions for use 


\title{
ANGULAR DISTRIBUTIONS OF ABSORBED DOSE OF BREMSSTRAHLUNG AND SECONDARY ELECTRONS INDUCED BY 18-, 28-, AND 38-MEV ELECTRON BEAMS IN THICK TARGETS
}

\author{
Masashi Takada ${ }^{\text {a* }}$,Kazuaki Kosako ${ }^{\text {b }}$, Koji Oishi ${ }^{\text {b }}$, Takashi Nakamura ${ }^{\text {b,c }}$, Kouichi Sato ${ }^{\text {d }}$, Takashi Kamiyama ${ }^{\mathrm{d}}$, \\ Yoshiaki Kiyanagi ${ }^{\mathrm{d}}$ \\ ${ }^{a}$ National Institute of Radiological Sciences, Chiba 263-8555, Japan, ${ }^{\text {b }}$ Institute of Technology, Shimizu \\ Corporation, Koto-ku, Tokyo 135-8530, Japan, ${ }^{\mathrm{c}}$ Cyclotron and Radioisotope Center, Tohoku University, Sendai \\ 980-8579, Japan, ${ }^{\mathrm{d}}$ Faculty of Engineering, Hokkaido University, Kita-ku, Hokkaido, 060-8628, Japan
}

\author{
Received on December , 2011, revised on, accepted on
}

\begin{abstract}
Angular distributions of absorbed dose of Bremsstrahlung photons and secondary electrons at a wide range of emission angles from 0 to 135 degrees, were experimentally obtained using an ion chamber with a $0.6 \mathrm{~cm}^{3}$ air volume covered with or without a build-up cap. The Bremsstrahlung photons and electrons were produced by 18-, 28-, and 38-MeV electron beams bombarding tungsten, copper, aluminum, and carbon targets. The absorbed doses were also calculated by multiplying simulated photon and electron energy spectra with simulated response functions of the ion chambers, using the MCNPX code. Our simulation results agree with the experimental results, within a factor of 2 , over wide ranges of emission angles, incident electron energies, and atomic numbers of targets. The angular distributions of absorbed doses at forward angles for incident energies are similar to those for targets. On the other hand, the absorbed doses at backward angles depend on target species. The dependences of absorbed doses on electron energy and target thickness were compared between the measured and simulated results, and showed good agreement. We also measured the attenuation profiles of absorbed doses of Bremsstrahlung beams at $\mathbf{0 , 3 0}$, and 135 degrees to the electron beam axis. Simulated attenuation profiles agree, in relative values, with the experimental results at incident electron energies and angles. To achieve further accuracy of calculation, angular absorbed-dose measurements are necessary for bremsstrahlung radiation dosimetry.
\end{abstract}

\section{INTRODUCTION}

Cancer constitutes the major human disease in modern society, and its impact on public health is very considerable. Indeed, radiotherapy such as irradiating tumor tissues with Bremsstrahlung from high-energy electron linear accelerators, is one of the most effective medical treatments for cancer. As of 2010, 931 electron linear accelerators were in operation in Japanese hospitals[1]; the number worldwide was about 7500[2]. These electron linear accelerator have used 4-20 MeV electron beams. To increase treatment efficiency and to treat cancer in a deeper body, higher energy accelerators have been required. High-energy X-rays offer several advantages over lower-energy photons.

The bremsstrahlung photons from the linear accelerator spread to various directions. Precise estimation of angular absorbed-dose distributions of Bremsstrahlung photons and secondary electrons are essential for the shielding of the linear accelerator room. Recently, the Monte Carlo codes have been used to evaluate the accurate optimum shielding design. The accuracy of angular distribution data of photon and electron beams produced from the target is very important, as a source data for shielding calculations.

In this work, the angular distributions of absorbed doses due to the Bremsstrahlung of electron beams

*Corresponding Author: m_takada@nirs.go.jp bombarding thick targets were measured with an ionization chamber. Our experiments cover wide ranges of emission angles, atomic numbers of target materials, and incident electron energies. Attenuation profiles of absorbed doses were experimentally obtained. Also, the experimental data are compared with Monte-Carlo simulation.

\section{EXPERIMENTS}

\section{Experimental Configuration}

Experiments were done using the $45 \mathrm{MeV}$ electron linear accelerator at Hokkaido University. The accelerator was operated at a nominal pulse repetition rate, $10 \mathrm{~Hz}$, and a nominal pulse width, $3 \mu \mathrm{s}$. Figure 1 shows the experimental setup. Electron beams were extracted through a $30-\mu$ m-thick Ti exit window. The target, which was isolated electrically from the target stand, was positioned at $20 \mathrm{~cm}$ downstream from the $\mathrm{Ti}$ window.

We experimentally obtained the angular distributions of absorbed doses at the $0,30,60,90,120$ and 135 degrees with respect to the beam axis, using ion chamber, covered with or without a build-up cap. In this study, the ion chamber without the build-up cap is called a bare ion chamber; however, the chamber covered with the cap is called a capped ion chamber. We used 


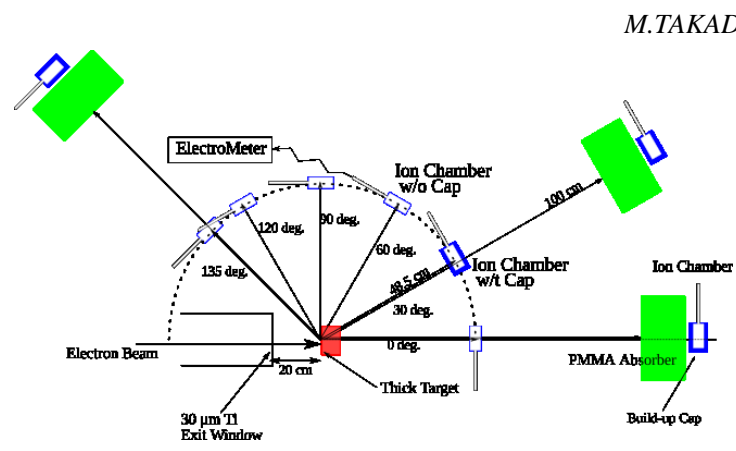

Figure 1. Experimental set-up of angular distribution and attenuation profile of absorbed doses.

the 4.55-mm-thick build-up cap, made of polymethyl methacrylate (PMMA).

The ion chamber was positioned around the target at $48.5 \mathrm{~cm}$ from the front surface of the target. For decrease of scatter electrons and photons, the ion chamber was positioned at $20 \mathrm{~cm}$ above the wooden stage. The side surface of the chamber was faced to the target, as shown in Fig. 1, at all angles. We acquired charge currents from the ion chamber using electrometer, Keithley 6527A.

Bremsstrahlung photons, secondary electrons, and photoneutrons were produced by several tens of electron beams bombarding the target. For measurement of attenuation length, attenuation profile of the absorbed dose was measured by putting PMMA plates with $20 \times$ $20 \mathrm{~cm}^{2}$ area, in front of the capped ion chamber, at the $100 \mathrm{~cm}$ from the target to the PMMA surface, as shown in Fig. 1. The attenuation profile was measured from 0 to $20 \mathrm{~cm}$ thickness at 0,30 and 135 degree with the respect to beam axis. The ion chamber was located at 2 $\mathrm{cm}$ downstream from back surface of the PMMA plat.

\section{Electron Energy Spectrum}

Electron energy spectra were measured from electron beam currents transported through a 7-mm-diameter collimator using a bending magnet, as shown in Fig. 2. Peak energies of 18,28 and $38 \mathrm{MeV}$ incident electron beams obtained were $18.2 \pm 6.3,27.7 \pm 4.1$, and $38.6 \pm 7.4 \mathrm{MeV}$, respectively, by fitting each peak with Gaussian distribution. Energy error indicates full widith half maximum (FWHM) of energy peak. These electron spectra were used as electron source spectra in the simulation. Nominal electron energies are labeled as 18 , 28 , and $38 \mathrm{MeV}$ from the peak energies in the energy spectra.

We experimentally obtained electron beam currents using a cylindrical copper Faraday cup $10.0 \mathrm{~cm}$ in length and $6.0 \mathrm{~cm}$ diameter, having a hole of $3.0 \mathrm{~cm}$ diameter and a depth of $5.0 \mathrm{~cm}$. The beam currents were measured, 6.0, 3.6, and $6.16 \mu \mathrm{A}$ for 18,28 , and $38 \mathrm{MeV}$ beam energies, respectively. Using this Faraday cup, simulated escaped electron flux from the Faraday cup were 7\%.

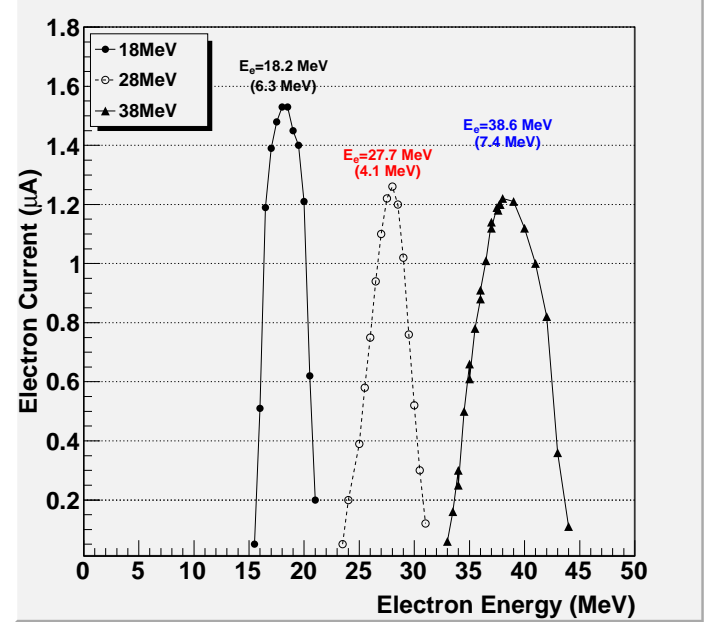

Figure 2. Measured electron energy spectra of 18, 28, and 38 $\mathrm{MeV}$. FWHM is in parentheses.

Almost electrons are escaped from side of the cup for electron-photon shower cascades.

\section{Spatial Distribution of Beam Profile}

We measured electron beam profiles at the target position, using a concentric-circle copper plates, as shown in Fig. 4. The plate covers an 49-mm-radius area. In the area, 3-mm-width rings cover the area within 31 $\mathrm{mm}$ in radius at $1 \mathrm{~mm}$ intervals; however, over $31 \mathrm{~mm}$ in radius 5-mm-width rings cover the area. The spatial distributions were measured from beam current at each electrically isolated ring. Measured beam profiles are shown in Fig. 3. Beam currents at the medium of ring widths normalized with ring area were plotted with $\mathrm{x}$-error bars. The error bars are equivalent to the ring widths.

Similar beam profiles of 18 and $28 \mathrm{MeV}$ electron beams were observed, however, the $38 \mathrm{MeV}$ electron beam profiles were wider than the other beams. The 18 and $28 \mathrm{MeV}$ beam profiles from 5 to $8 \mathrm{~mm}$ radii are contributed from 27 to $29 \%$ to the center; however, the 38 $\mathrm{MeV}$ beam profile was $58 \%$ of the beam center. These beam profiles were used for spatial electron distribution in the simulation.

\section{Targets}

For the wide range of atomic number of tagets from 6 to 74 , we selected these targets: $1-\mathrm{cm}$-thick tungsten, 2 and $3-\mathrm{cm}$-thick copper, 4 and 6-cm-thick aluminum and 10.5-mm-thick graphite. The sizes of $\mathrm{W}, \mathrm{Cu}$ and $\mathrm{Al}$ targets were $4 \mathrm{~cm}$ high and $4 \mathrm{~cm}$ wide; however, the size 


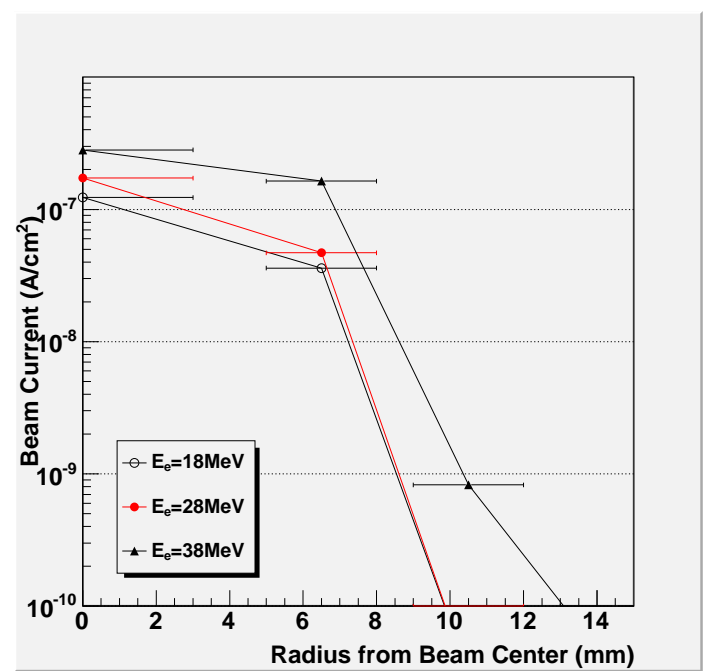

Figure 3. Measured electron beam profiles, from 18 to $38 \mathrm{MeV}$ electron beams. $\mathrm{X}$-axis error bars are equivalent to ring widths.

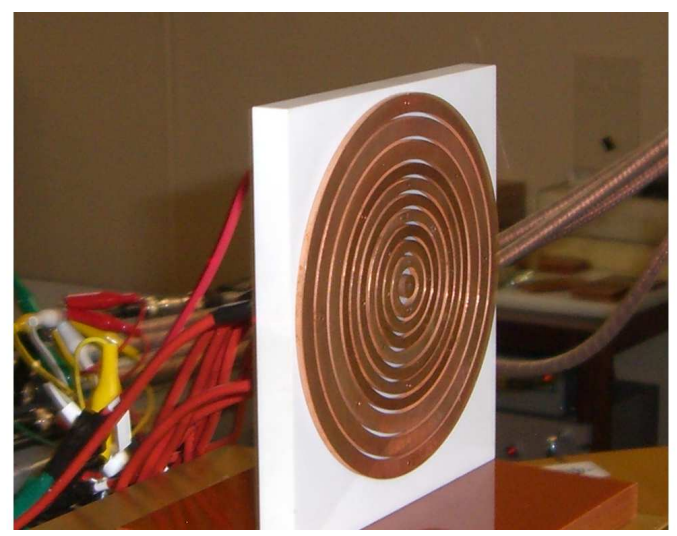

Figure 4. Picture of concentric-circle copper beam profile monitor.

of $\mathrm{C}$ target was $23 \mathrm{~cm}$ high and $20 \mathrm{~cm}$ wide. These target sizes and densities are listed in Table 1.

Ranges of $38 \mathrm{MeV}$ electrons in these targets [4] are tabulated in the table. For comparison of electron ranges and target thickness, electron ranges [4] are plotted in Fig. 5. The thicknesses of the tungsten, copper, and carbon targets were enough to stop $38 \mathrm{MeV}$ electron beams in the targets; however, the 4-cm-thick aluminum target is thinner than the ranges of the 28 and $38 \mathrm{MeV}$ electrons. The 6-cm-thick aluminum target was added to stop $28 \mathrm{MeV}$ electron beams. Since the range of 38 $\mathrm{MeV}$ electrons in aluminum $(6.39 \mathrm{~cm})$ is longer than the 6 -cm-thick aluminum target, $22 \%$ of electrons are transmitted through the aluminum target. Copper targets with two thicknesses are used to measure target-thickness
Table 1. Target configuration

\begin{tabular}{c|ccccc}
\hline & $\begin{array}{c}\text { Atomic } \\
\text { Number }\end{array}$ & $\begin{array}{c}\text { Density } \\
\left(\mathrm{g} / \mathrm{cm}^{3}\right)\end{array}$ & $\begin{array}{c}\text { Thickness } \\
(\mathrm{cm})\end{array}$ & $\begin{array}{c}\text { Size } \\
\left(\mathrm{cm}^{2}\right)\end{array}$ & $\begin{array}{c}\text { Range } \\
(\mathrm{cm})\end{array}$ \\
\hline $\mathrm{W}$ & 74 & 19.3 & 1.0 & $2 \times 2$ & 0.69 \\
$\mathrm{Cu}$ & 29 & 8.96 & 2 & $2 \times 2$ & 1.76 \\
$\mathrm{Cu}$ & 29 & 8.96 & 3 & $2 \times 2$ & 1.76 \\
$\mathrm{Al}$ & 13 & 2.70 & 4 & $2 \times 2$ & 6.39 \\
$\mathrm{Al}$ & 13 & 2.70 & 6 & $2 \times 2$ & 6.39 \\
$\mathrm{C}$ & 6 & 1.75 & 10.5 & $23 \times 20$ & 11.0 \\
\hline
\end{tabular}

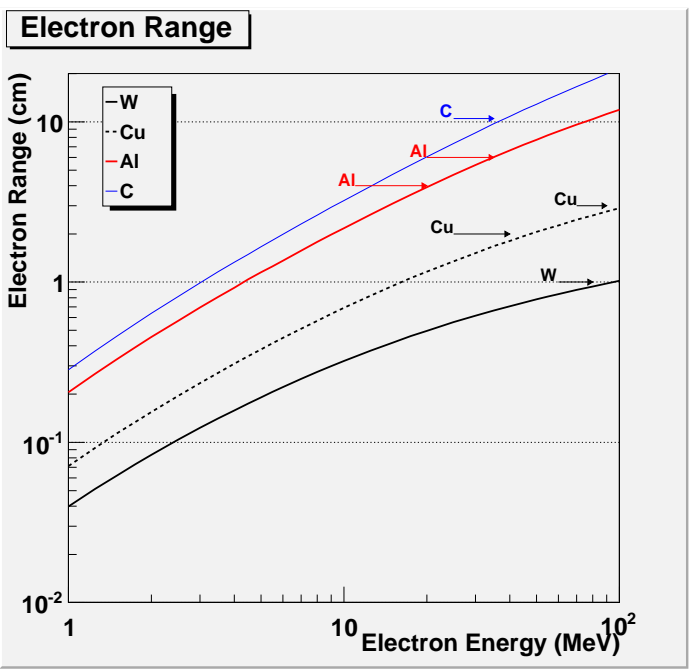

Figure 5. Electron range in $\mathrm{W}, \mathrm{Cu}, \mathrm{Al}$, and $\mathrm{C}$. Arrows indicate target thicknesses [4].

dependence of absorbed doses. Electron supresser was not put at the target to measure escaped electrons.

\section{Ion Chamber}

\section{Chamber Configuration}

We measured the absorbed doses using a farmer-type ionization chamber (Type 30010, PTW-Freiburg, Germany) with $0.6 \mathrm{~cm}^{3}$ sensitive volume, as shown in Fig. 6 . The chamber has a $0.355-\mathrm{mm}$-thick PMMA wall and an aluminum central electrode with $1.1 \mathrm{~mm}$ diameter. Voltage of $+400 \mathrm{~V}$ was applied to the chamber.

Calibrated chamber sensitivity was $50 \mathrm{mGy} / \mathrm{nC}$ for ${ }^{60} \mathrm{Co}$ photons at the national standard laboratory in the national institute of advanced industrial science and technology in Tsukuba, Japan. At the calibration, the chamber was covered with a 4.55-mm-thick PMMA build-up cap to satisfy charged-particle equilibrium. We obtained the absorbed doses from measured charge with the ion chamber, using this calibration factor. 
(a) Ion Chamber

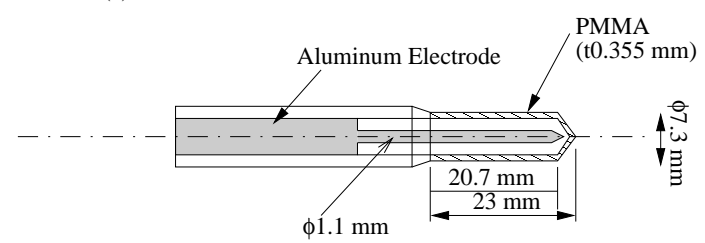

(b) PMMA Build-up Cap

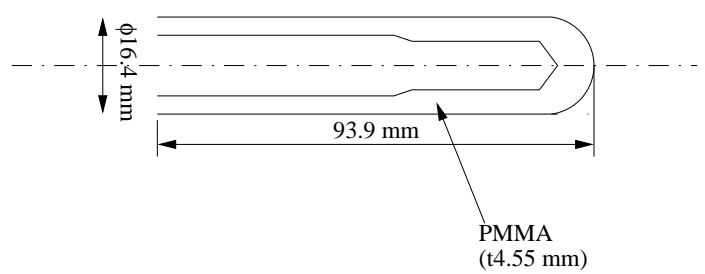

Figure 6. Configuration of ion chamber.

\section{Chamber Response}

Response functions of the ion chamber were simulated using the MCNPX code [3] for photons and electrons up to $50 \mathrm{MeV}$. Figure 7 shows simulation geometries for the bare or PMMA-covered ion chamber. Photons and electrons are incident to the side surface of the ion chamber. Deposited energies in air (tally score region), shown in Fig. ?? are scored using the pulse-height tally (tally 8) in the MCNPX code. The absorbed doses in air per unit electron and photon fluences are plotted, as a function of particle energy, in Fig. 8.

Electron response of the bare or capped ion chamber increases sharply at 0.2 and $1 \mathrm{MeV}$, respectively, as indicated by arrows. This different threshold energies are induced from absoprtion of electrons below 1 $\mathrm{MeV}$ in the build-up cap. Maximum photon energies for satifying charged-particle equilibrium are 0.5 and 2.5 $\mathrm{MeV}$ for bare and capped ion chambers, respectively, indicated with arrows. Above these energies, the chamber response decreases due to a lack of charged-particle equilibrium.

Electron response of cylindrical ion chamber is dependent on incident angles for different path length of incident electron in the chamber. We simulated deposited energies with incident electron angles. The simulation indicates the angular response was within $7 \%$ dispersion for $15 \mathrm{MeV}$ electrons and less than $1 \%$ dispersion for lower electron energies.

\section{Ion-Chamber Sensitivity Correction}

The ion chamber is sensitive to atmospheric temperature and pressure. Both the values were measured around the chamber through the experiments. The chamber sensitivity to temperature and pressure is corrected using a

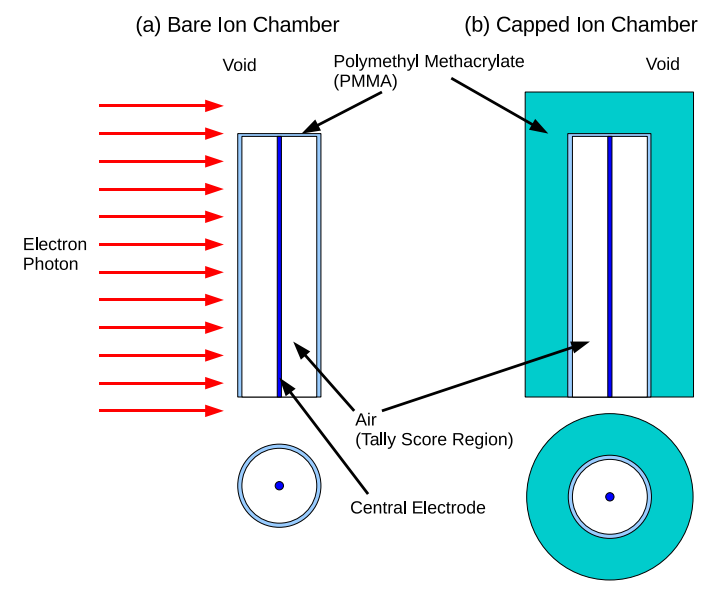

Figure 7. MCNPX simulation geometry for ion-chamber response functions to electrons and photons: (a) bare or (b) PMMA covered ion chamber.

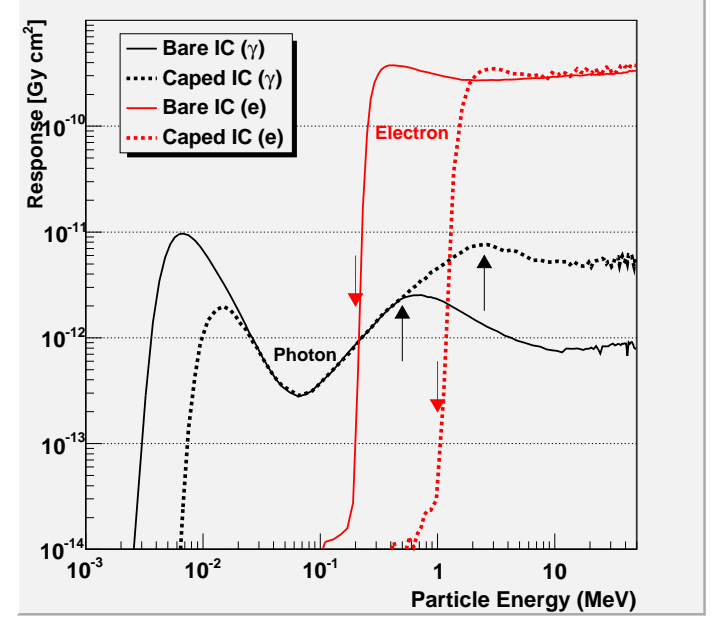

Figure 8. Simulated response functions of bare (solid line) or caped (broken line) ion chamber to electrons and photons.

factor $f_{t p}$

$$
f_{t p}=\frac{T_{0}+T}{T_{0}} \cdot \frac{P_{0}}{P}
$$

where $T_{0}$ and $P_{0}$ are standard temperature $(273.15$ $\mathrm{K})$ and pressure $\left(1013 \times 10^{5} \mathrm{~Pa}\right)$, respectively. $T$ and $P$ are air temperature and pressure in the chamber, respectively.

Under intense radiation fields, free electrons produced in the chamber can recombine with positive ions, and this phenomenon induces underestimation of the absorbed doses. We corrected recombination of electrons using the charge collection efficiency $f_{r}$. [5] The 
ANGLE ABSORBED-DOSE DISTRIBUTION OF BREMSSTRAHLUNG

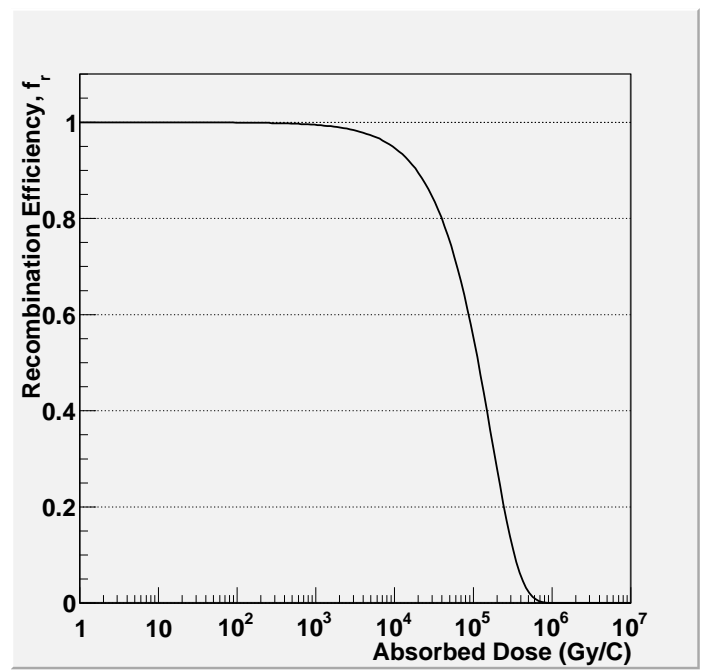

Figure 9. Charge collection efficiency as a function of absorbed dose per incident electron beam charge at target.

collection efficiency $f_{r}$ is defined as ratio of collected charges to liberated ones, given as follows:

$$
\begin{aligned}
f_{r} & =\frac{\ln \left(1+v_{c}\right)}{v_{c}} \\
v_{c} & =\frac{\mu r d^{2}}{V} \\
d^{2} & =(a-b) \frac{a / b+1}{a / b-1} \times \frac{\ln (a / b)}{2},
\end{aligned}
$$

where $\mu$ is a constant for any particular gas independent of chamber geometry, $3.02 \times 10^{10} \mathrm{~V} / \mathrm{mC}, V$ is the voltage applied to the ion chamber, $r$ is the charge density liberated per pulse, and $a$ and $b$ are the outer and inner electrode radii, respectively. The charge density $r$ was calculated from absorbed dose (Gy) in the ion chamber per beam pulse. The collection efficiency $f_{r}$ is calculated, as shown in Fig. 9, as a function of absorbed dose per incident beam current in $(\mathrm{Gy} / \mathrm{C})$. Using this curve, we corrected the charge recombination to obtain the absorbed doses in air.

\section{Uncertainties in Experimental Data}

We considered several uncertainties in the measurement, as shown in Table 2. We measured charges from the chamber several times under the same conditions. Charges from the chamber ("Chamber Charge" in the table) dispersed within $0.5 \%$ (0.9951 to 1.0041). The fluctuation of the electron beam current ("Beam Curr.") was 0.1 to $0.2 \mu \mathrm{A}(3-6 \%)$. The temperature and pressure ("Temp. Press.") varied during the experiments, from 0.9986 to 1.0013 and from 0.9971 to 1.0030 , as the ratio
Table 2. Uncertainty of absorbed-dose measurement.

\begin{tabular}{c|ccccc|c}
\hline $\begin{array}{c}\text { Energy } \\
(\mathrm{MeV})\end{array}$ & $\begin{array}{c}\text { Chamber } \\
\text { Charge }\end{array}$ & $\begin{array}{c}\text { Beam } \\
\text { Curr. }\end{array}$ & $\begin{array}{c}\text { Temp. } \\
\text { Press. }\end{array}$ & BG & Geom. & Total \\
\hline 18,38 & $0.5 \%$ & $3 \%$ & $0.4 \%$ & $0.1 \%$ & $2 \%$ & $3.7 \%$ \\
28 & $0.5 \%$ & $6 \%$ & $0.4 \%$ & $0.1 \%$ & $2 \%$ & $6.4 \%$ \\
\hline
\end{tabular}

of absorbed doses to their average values, respectively. Photons emitted from surrounding materials activated by photoneutrons ("BG") were measured to be less than $0.02 \mathrm{mGy}$ before and after beam irradiation. Uncertainties of distance from the target and angle of the ion chamber ("Geom.") were considered $0.5 \mathrm{~cm}$ and 1.7 degree, respectively, from the ion chamber size in this study. This uncertainty was estimated to be $2 \%$.

Microwave absorbed dose was detected to be 0.35 mGy at chamber position. This microwave was produced from the electron linear accelerator before electron irradiation to the target. To remove the microwave noise, absorbed doses were measured after complete warm up of electron gun in the accelerator for each measurement. No microwave noise was not included in our measurements. Scattered electrons and photons produced from surrounding materials were simulated to be negligible.

Photoneutrons produced at the targets were observed by Kosako [7]. These photoneutrons show smaller fluxes than electron and photon fluxes. But, the ion chamber is sensitive to neutrons because of the hydrogen nucleus in the chamber wall, PMMA. We simulated the neutron response of the ion chamber from 0 to $50 \mathrm{MeV}$ using the MCNPX code [3], as shown in Fig. 10. The nuclear data libraries selected were JENDL/HE [8] above $20 \mathrm{MeV}$ and LA150 [9] libraries below $20 \mathrm{MeV}$. Fig. 10 plots deposited energies in the cavity region by proton, deuteron, triton, alpha and $\mathrm{He}-3$ produced from the neutron reaction with the PMMA wall, and summation of their deposited energies. Below $20 \mathrm{MeV}$, proton deposition is main contribution; however, over $20 \mathrm{MeV}$, main contribution is changed to alpha. But, the neutron responses were $10^{-5}$ smaller than the photon response, $5 \times 10^{-13}$ $\left[\mathrm{Gy} /\left(\mathrm{n} / \mathrm{cm}^{2}\right)\right]$. Neutron detection was negligible in our measured doses.

Finally, the total uncertainties we obtained were $3.7 \%$ for 18 and $38 \mathrm{MeV}$ electron beams, and $6.4 \%$ for 28 $\mathrm{MeV}$. In this study, beam currents induced the largest errors.

\section{SIMULATION OF SECONDARY ELECTRON AND PHOTON}

\section{Monte Carlo Code}

We simulated the radiation field produced from electromagnetic showers using the MCNPX Monte Carlo transport code system [3]. For photons, the code takes 


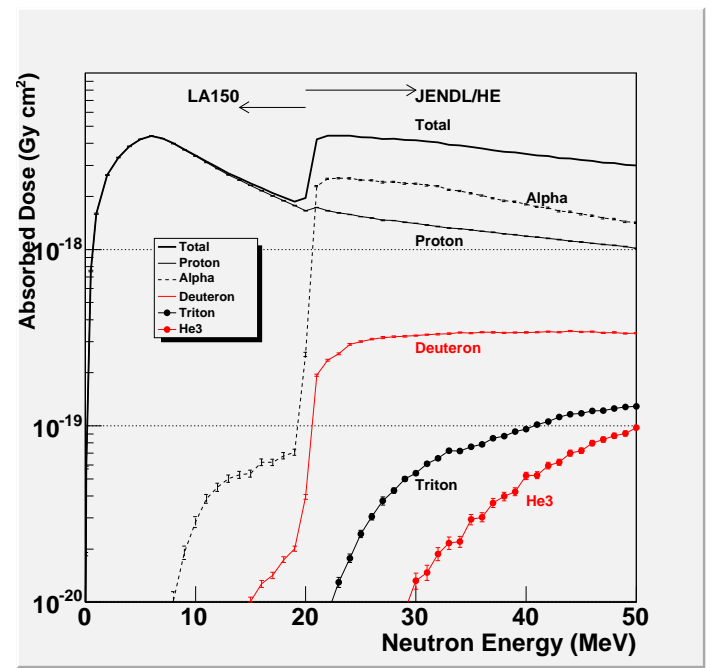

Figure 10. Simulated neutron response function of ion chamber of several secondary particles created by neutrons, using MCNPX code with nuclear data libraries, LA150 below 20 $\mathrm{MeV}$ and JENDL/HE above $20 \mathrm{MeV}$.

account of incoherent and coherent scattering, the possibility of fluorescent emission after photoelectric absorption, and Bremsstrahlung effect. The electron and photon interaction data libraries used were EL03 [10] and MCPLIB04 [11], respectively.

The energy indexing with the Integrated TIGER Series (ITS) improves electron transport in the MCNPX simulation. The default mode of MCNP is reported to lead to serious errors when the dose distribution is calculated around beta sources due to an error in the electron transport [12]. In this study, the ITS energy index is applied, whereas other parameters are used as defaults.

\section{Benchmark Simulation}

First, we made benchmark tests of our simulation using the Bremsstrahlung photon energy spectra. Faddegon et al. [13] measured the Bremsstrahlung photons produced by $15 \mathrm{MeV}$ electrons bombarding a beryllium target $11.67\left(\mathrm{~g} / \mathrm{cm}^{2}\right)$ thick, an aluminum target $9.74\left(\mathrm{~g} / \mathrm{cm}^{2}\right)$ thick, and a lead target $9.13\left(\mathrm{~g} / \mathrm{cm}^{2}\right)$ thick. They measured the photon energy spectra using a $\mathrm{NaI}(\mathrm{Tl})$ scintillator collimated with $\mathrm{Pb}$ blocks at $0,10,30,60$, and 90 degrees to the electron beam axis.

Figure 11 compares our simulated and their experimental photon energy spectra. The simulated spectra agree well with the measured spectra within experimental errors at wide ranges of emission angles and incident electron energies for three targets. Based on these results, we simulated the Bremsstrahlung photon spectra measured for various cases in this study. Electron transport in the code was confirmed with electron-photon cascade, but this simulation does not confirm electron spectra and deposited energies.

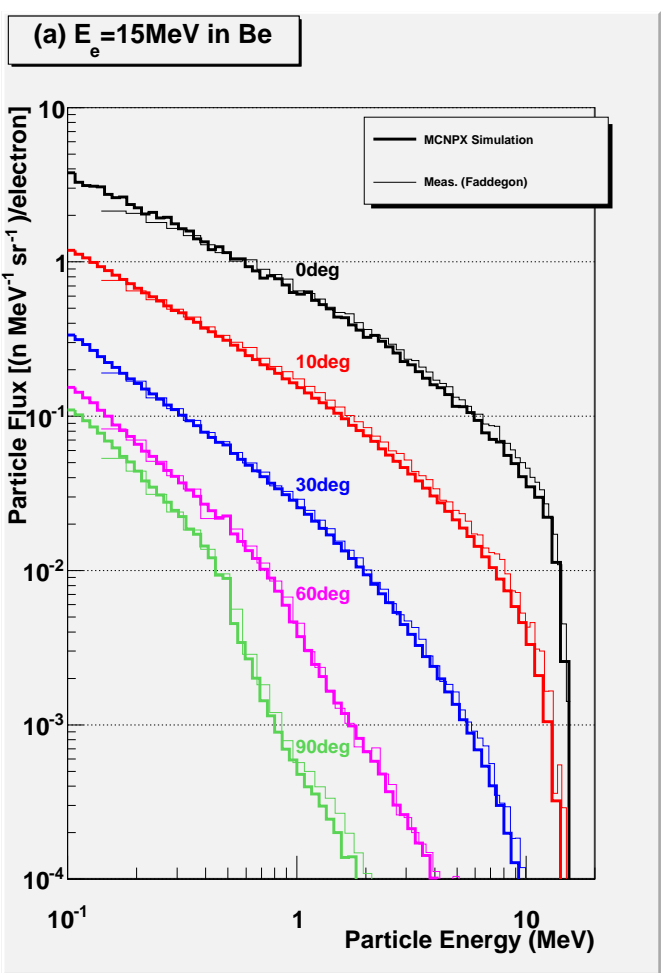

Figure 11. Simulated angular photon energy spectrum (thick solid line), from 0 to 90 degrees, produced by $15 \mathrm{MeV}$ electrons bombarding $\mathrm{Be}(\mathrm{a}), \mathrm{Al}(\mathrm{b})$, and $\mathrm{Pb}(\mathrm{c})$, compared with the experimental photon energy spectrum (thin solid line) in Ref. [13].

\section{Angular Distributions}

Figure 12 shows simulation geometry of angular-dose distributions of Bremsstrahlung photons and secondary electrons produced by electron beams bombarding the target. The simulation considers Ti beam exit window, air, beam pipe and target stand. Air region was in the shape of a semicircular column with a $50 \mathrm{~cm}$ radius and $20 \mathrm{~cm}$ in height, for save of CPU time. Photons and electrons were transported down to $1 \mathrm{keV}$. The energy spectra and beam profiles of incident electrons in the simulation are referred from the experimental results, as shown in Figs. 2 and 3. The ion chamber was replaced with air-filled sphere cell with $1.2 \mathrm{~cm}$ radius for save of CPU time. This sphere cell was positioned from 0 to 180 degrees to the electron beam axis at 15-degree intervals. Deposited energies in the sphere cells were scored using the crossing cell tally of MCNPX code (tally 4). 

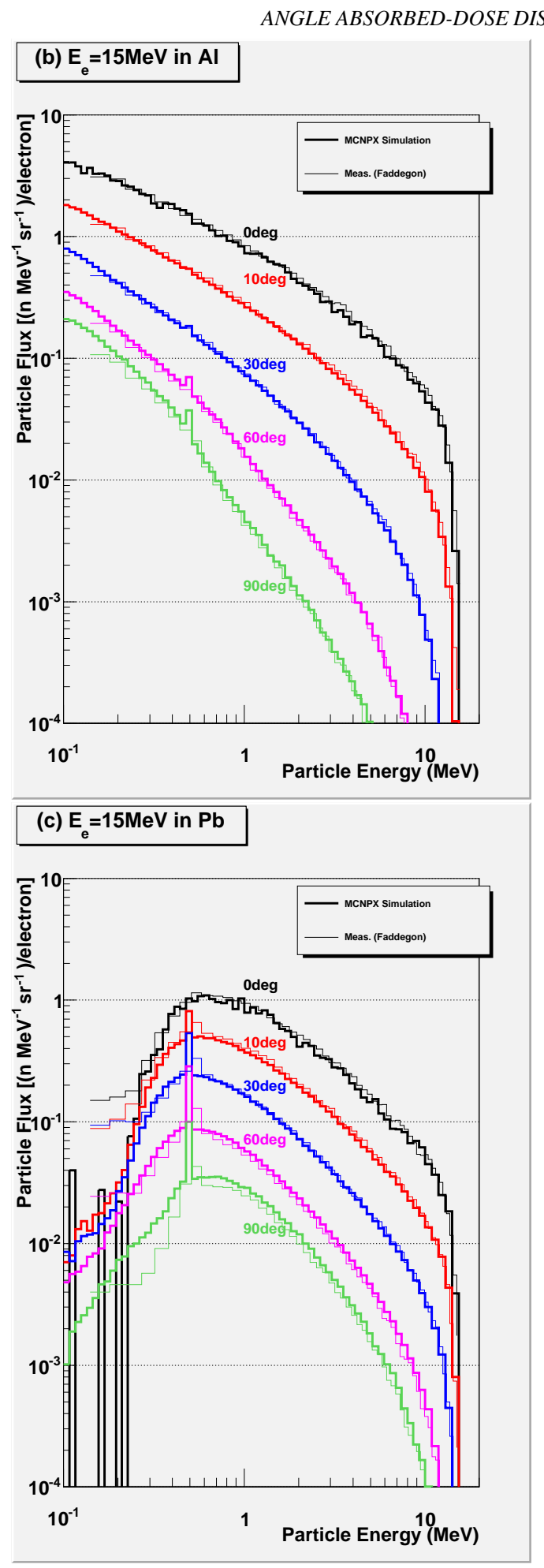

Figure 11. Continued

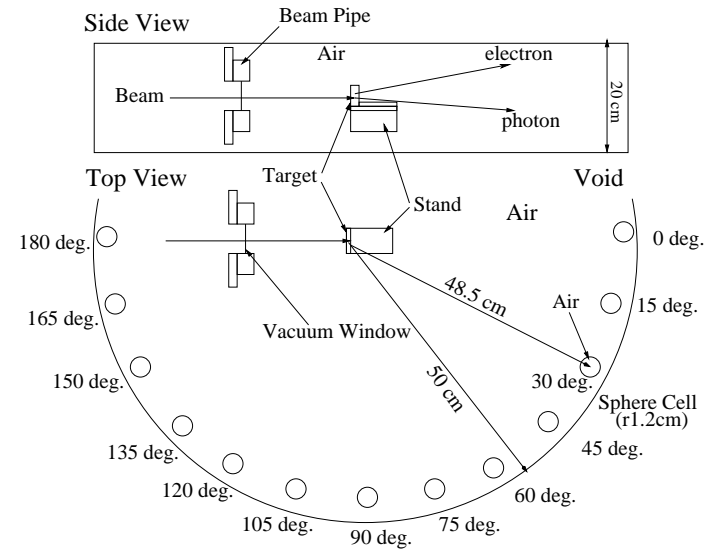

Figure 12. MCNPX simulation geometry for photons and electrons produced by electron beams bombarding target.

Finally, we obtain absorbed doses $D$ around the target, by multiplying the energy spectra of electrons and photons $\phi_{e, \gamma}(E)$ and the chamber responses $R_{e, \gamma}(E)$ given in Fig. 8, as described in Eq. 3,

$$
D=\sum_{e, \gamma} \sum_{E} \phi_{e, \gamma}(E) \times R_{e, \gamma}(E)
$$

\section{Attenuation Profile}

With several PMMA thicknesses, ion-chamber responses were simulated at $2 \mathrm{~cm}$ downstream from the PMMA plates $20 \mathrm{~cm}$ high and $20 \mathrm{~cm}$ wide, as shown in Figure 13. Photon and electron energies ranges from 0 to 50 $\mathrm{MeV}$, and the PMMA ranges from 0 to $20 \mathrm{~cm}$ in thickness. Electron beam covers PMMA plate area. Same process was done as the chamber response simulation for scoring deposited energy.

Maximum photon energies satisfying charged-particle equilibrium, as indicated by arrows increase with PMMA thickness: 2, 5 and $10 \mathrm{MeV}$ electron energies for 0,1 and $2 \mathrm{~cm}$ in thickness, respectively. On the other hand, the electron responses increase sharply at 1.1, 2.7, 4.5 and $11 \mathrm{MeV}$ for $0,1,2,5$ and $10 \mathrm{~mm}$ in thickness, respectively. Attenuation profiles were obtained multiplying these response function, as shown in Fig. 13 with secondary photon and electron energy spectra. The same calculation in Eq. 3 was used.

\section{RESULTS AND DISCUSSION}

\section{Angular Distribution}

\section{Bare Ion Chamber}

Figure 14 compares experimental (circles) and simulated (solid lines) angular distributions of absorbed doses with three incident electron energies $(18,28$, 

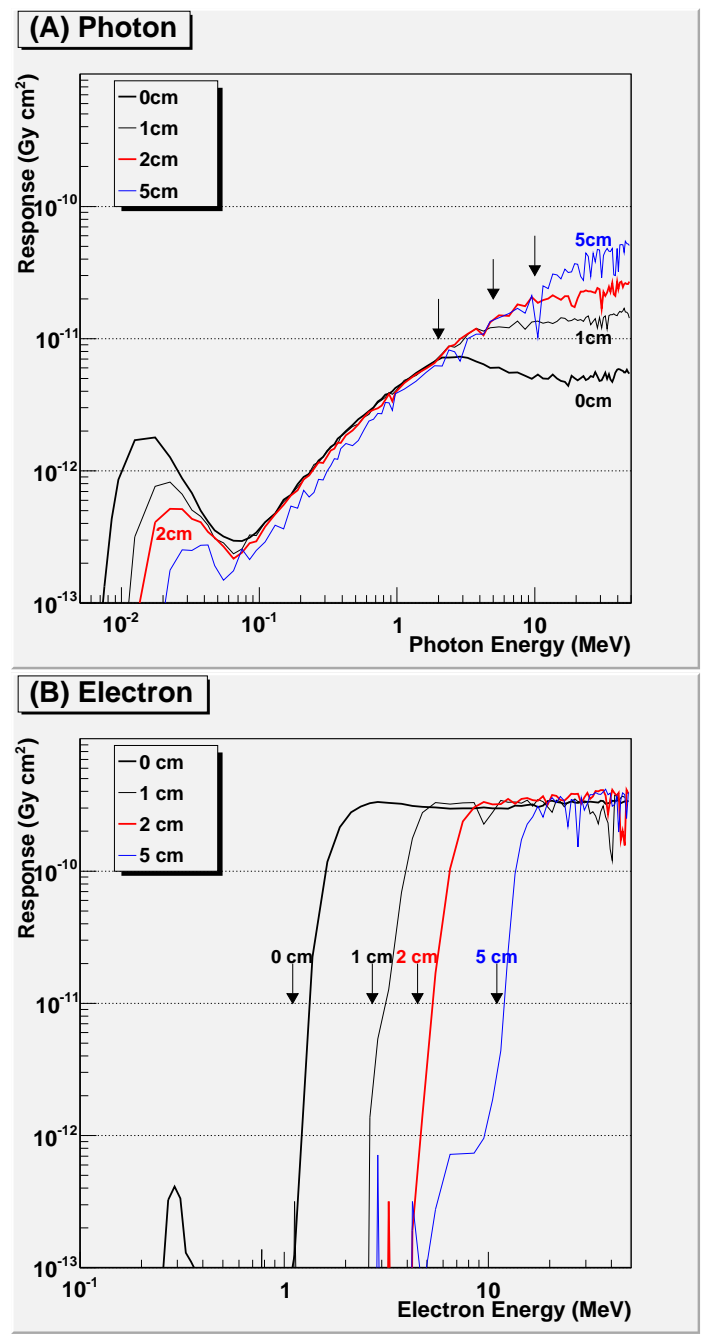

Figure 13. Simulated ion-chamber responses at $2 \mathrm{~cm}$ downstream from PMMA plates with several thicknesses for photons (A) and electrons (B).

and $38 \mathrm{MeV}$ ) and four targets [(a)tungsten, (b)copper, (c)aluminum and (d)carbon]. Angles are plotted as a laboratory system at the horizontal axis, and absorbed doses per incident electron beam current are plotted in $\mathrm{Gy} / \mathrm{C}$ unit at the vertical axis. The measured absorbed doses are corrected for atmospheric temperature and pressure, as well as for the recombination of created electrons and ions in the chamber. For comparison, original and recombination-corrected absorbed doses are plotted as open and filled circles, respectively. The simulated absorbed doses are separated into photon and electron distributions, shown as solid line with cross mark and solid line with open circles, respectively.
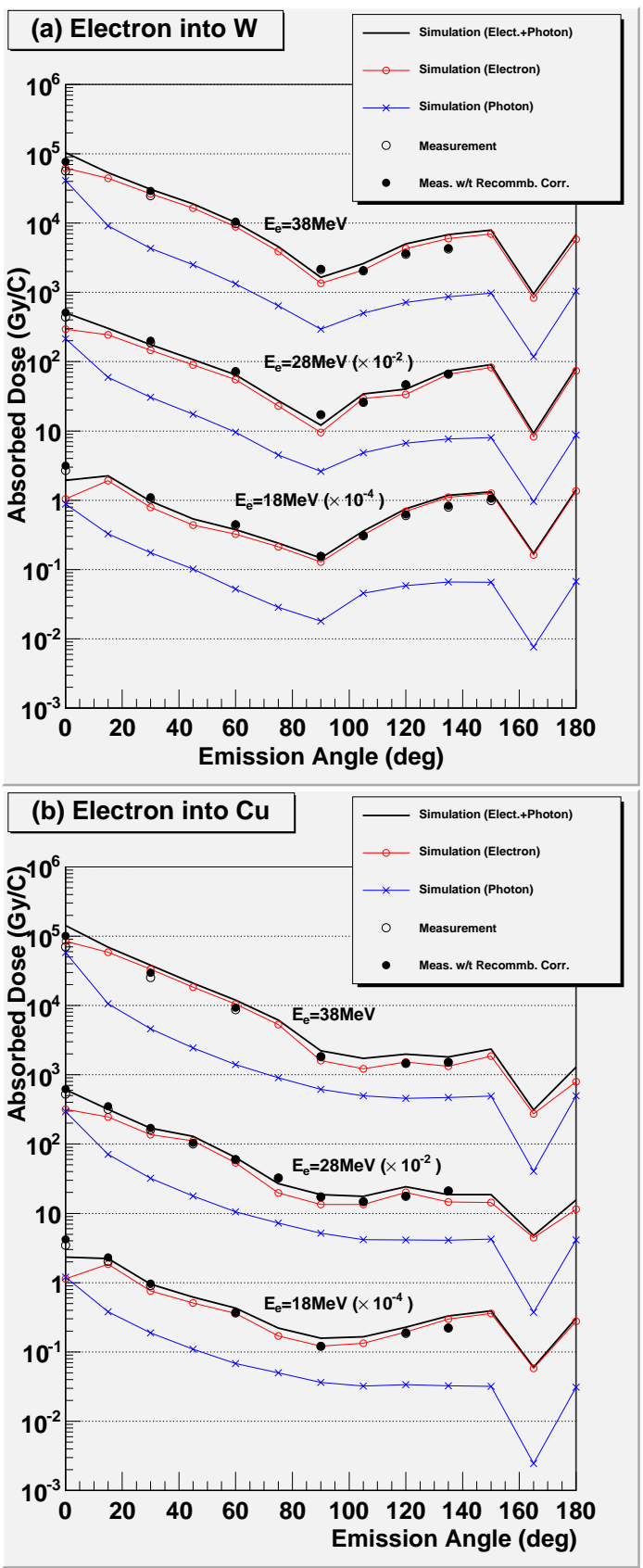

Figure 14. Measured and simulated angular distributions of absorbed doses, produced by 18,28 , and $38 \mathrm{MeV}$ electron beams into $\mathrm{W}(\mathrm{a}), \mathrm{Cu}(\mathrm{b}), \mathrm{Al}(\mathrm{c})$, and $\mathrm{C}(\mathrm{d})$. The measured absorbed doses, corrected with and without electron-ion recombination are plotted as filled and open circles, respectively. The simulated absorbed doses (solid line) was summed with electron (solid line with open circle) and photon doses (solid line with cross mark). The angular distributions produced by 18 and $28 \mathrm{MeV}$ electron beams were multiplied by 0.01 and 0.0001 , respectively. 
ANGLE ABSORBED-DOSE DISTRIBUTION OF BREMSSTRAHLUNG
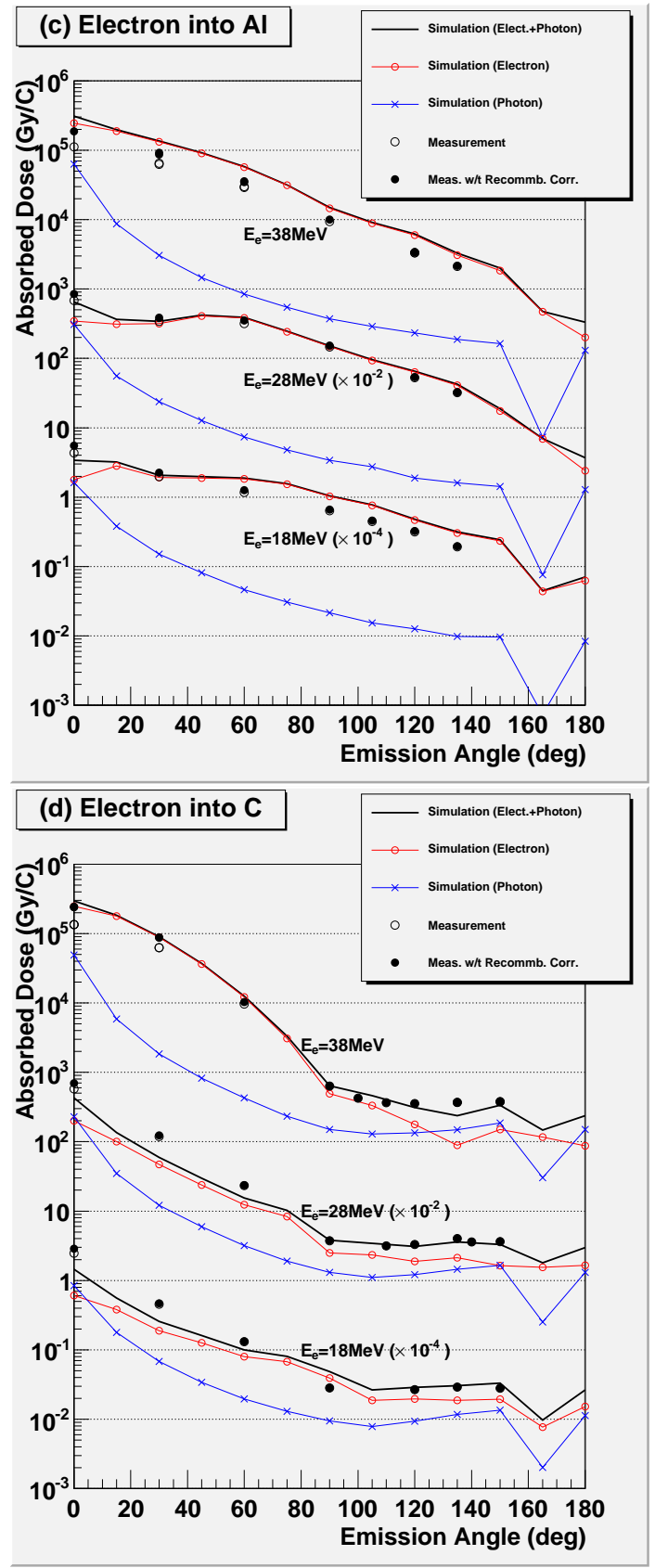

Figure 14. Continued

Our experimental results agree well with the simulation across wide ranges of atomic numbers of targets, emission angles, and incident electron energies. At the forward angles, dose ratios of calculation to experiment $(\mathrm{C} / \mathrm{E})$ on $\mathrm{W}$ and $\mathrm{Cu}$ are within factor 1.29 , except some points. At the 0 degree the $\mathrm{C} / \mathrm{E}$ are 1.41 and 0.55 for 34 and $18 \mathrm{MeV}$ electron beam, respectively. At the backward angles, $\mathrm{C} / \mathrm{E}$ on $\mathrm{W}$ and $\mathrm{Cu}$ are within factor 1.56. Large simulated doses than the experiment at 34 $\mathrm{MeV}$ are induced by overestimation of electron doses, and smaller doses of simulation are induced by underestimation of electron doses. Since we simulated the Bremsstrahlung photon energy spectra produced by 15 $\mathrm{MeV}$ electron beams at several targest, as shown in Fig. 11, electron simulation may induce these differences. $\mathrm{C} / \mathrm{E}$ values at the backward angle are incuded from uncertainty of electron-electron transports in the simulation.

For light targets of aluminum and graphite, the agreement between the simulation and experiment is worse than the hevay tagets of $\mathrm{W}$ and $\mathrm{Cu}$. For aluminum target at $28 \mathrm{MeV}$ beam, $\mathrm{C} / \mathrm{E}$ is within 1.3 from 0 to 135 degree. For graphite target, $\mathrm{C} / \mathrm{E}$ is within 1.2 at $38 \mathrm{MeV}$ from 0 to 150 degree, and $\mathrm{C} / \mathrm{E}$ is within 1.2 at $28 \mathrm{MeV}$ from 90 to 135 degree and $18 \mathrm{MeV}$ from 120 to 150 degree. But, other electron energies and angles shows large $\mathrm{C} / \mathrm{E}$, from 1.3 to 2.1 . At the 0 degree, similar ratios are obtained as heavy targets. $\mathrm{C} / \mathrm{E}$ are 1.2 and 0.5 for 38 and $18 \mathrm{MeV}$, respectively. At $38 \mathrm{MeV}$ beam in aluminum target the measured doses are around 50\% larger than the simulation. In the simulated doses, electron components dominated from 48 to $60 \%$ at the 0 degree. At other angles the electron doses dominated to be 70 98\%. In our measurement, electron component is main contribution.

This difference between experiment and simulation is induced from electron transport [14]. Their calculation using EGS5 and ITS3.0 codes indicate agreement within 1.5 to the experiment below $14 \mathrm{MeV}$ electron beam. For heavy and light targets, their C/Es are within 1.1 and 1.5 , respectively. Low-Z targets induce large dispersion beweeen simulation and experiment. They concluded that this difference has not yet been clarified. Our difference between the simulated and experimental results is introduced from the electron transport in the Monte Carlo code. Photon and electron fields have forward dose peaks and extend to backward angles. Although similar angular distributions were obtained at the forward angles, the angular distributions at the backward angle depend on the target species. A target with a large atomic number shows an increase in absorbed doses with emission angles, whereas a target with a small atomic number shows a dose decrease with the angles. At 90 and 165 degrees, the calculated absorbed doses decrease sharply because of electron absorption in the targets and the beam duct, respectively.

The simulated absorbed doses for the metal targets $(\mathrm{W}, \mathrm{Cu}$, and $\mathrm{Al})$ agree with the measurement within a factor of 1.8. For the carbon target, the absorbed doses are simulated within a factor of 2 . The measured result of $28 \mathrm{MeV}$ electron beam bombarding $\mathrm{W}, \mathrm{Cu}$, and $\mathrm{Al}$ targets shows better agreement with the simulation (ratio of calculation to experimental result, $\mathrm{C} / \mathrm{E}=0.66$ to 1.36 ) 


\section{M.TAKADA ET AL}

than those of the 18 and $38 \mathrm{MeV}$ electron beams. In the radiation fields produced by tens of $\mathrm{MeV}$ electron beams, the electron dose components at 0 degrees are primarily in the range of $45-60 \%$. For aluminum and carbon targets bombarded by $34 \mathrm{MeV}$ electron beams, the electron components show a much larger value, $80 \%$. At larger angles, the electron components constitute $70-98 \%$ of the total absorbed doses; however, the electron ratio for the carbon target is smaller, 30 $65 \%$, than for the other. Photon and electron fields have forward dose peaks and extend to backward angles. Although similar angular distributions were obtained at the forward angles, the angular distributions at the backward angle depend on the target species. A target with a large atomic number shows an increase in absorbed doses with emission angles, whereas a target with a small atomic number shows a dose decrease with the angles. At 90 and 165 degrees, the calculated absorbed doses decrease sharply because of electron absorption in the targets and the beam duct, respectively.

Based on the excellent agreement among the Bremsstrahlung photon energy spectra produced by $15 \mathrm{MeV}$ electron beams at several targets, as described in previous section, "Benchmark Simulation", the differences between our experimental and simulated results may be attributable to electron transport in the simulation. Especially for the experimental results of the aluminum target bombarded by the $38 \mathrm{MeV}$ electron beam, the dose difference was large $(\mathrm{C} / \mathrm{E}=1.5$ to 1.8$)$, as shown in Fig. 14-(c). This large discrepancy may be attributable to the thinness of aluminum target. At the $38 \mathrm{MeV}$ electron beam, $22 \%$ of electrons were transmitted through the aluminum target. At the $28 \mathrm{MeV}$ electron, which stops in the 6-cm-thick aluminum target, the simulated result agrees with the experimental result within a factor of 1.5. The absorbed-dose distribution of the $18 \mathrm{MeV}$ electron shows a different shape between the simulation and the experiment. For a carbon target bombarded by the 18 $\mathrm{MeV}$ electron beam, the simulation was underestimated as 0.5 to 0.6 of the experimental values at forward angles. Since electrons are charged particles with relatively low mass, accurate Monte Carlo simulation of their transport through matter has proven notoriously difficult, especially at lower energies. Electron transport of multiple-scattering and energy-loss processes could induce errors in the electron doses.

\section{Electron Step Size}

In this radiation field, absorbed doses are composed mainly of electrons. However, at the forward angle, 0 degrees, the proportional of photons is large. Electron transport in the simulation may be strongly affected by the conditions of the target, ion chamber, and surrounding materials; that is, energy deposition is sensitive to electron step size in the Monte Carlo simulation at the target and the chamber wall. The step size is explained as the number of sub steps per energy step. Energy deposition in the build-up capped ion-chamber for 1 and 10 $\mathrm{MeV}$ electrons was simulated using several step sizes. For high-energy electrons of 5 and $10 \mathrm{MeV}$, dispersion of about $2 \%$ of the absorbed doses was obtained using the default step size, although for $1 \mathrm{MeV}$ electrons, the dispersion was larger, about $20 \%$, due to the large stopping power of the materials. Here, we chose the default step sizes, which are 12 for tungsten, 7 for copper, 5 for aluminum, and 3 for carbon.

\section{Build-up Capped Ion-Chamber}

Figure 15 compares the angular distributions of absorbed doses between measurement using the build-up capped ion-chamber and the MCNPX simulations. The MCNPX simulations of the absorbed doses agree with the measurement within a factor of 1.75 at wide ranges of emission angles and incident electron energies for two targets $(\mathrm{C} / \mathrm{E}=0.75-1.61$ for $\mathrm{W}$, and $0.73-1.75$ for $\mathrm{Cu})$. Large $\mathrm{C} / \mathrm{E}$ values are obtained at 0 degrees.

Figure 16 shows the ratio of absorbed doses measured using the ion chamber covered with or without the build-up cap for the tungsten and copper targets. At the forward angles from 0 to 30 degrees, the ratio is over 1.0. On the other hand, at larger angles, the ratio is less than 1.0. At 0 degrees, the dose ratio increases by the buildup effect of photons. At large angles, over 45 degrees, since electrons were shielded in the PMMA cap, the electron absorbed doses decreased with the cap. From the dose ratio, photons are found to contribute to the 0 degree dose. Meanwhile, at over 60 degrees, electrons are dominant in the absorbed doses. At the backward angle, the dose ratio of the tungsten target is larger than that of the copper target.

\section{Target Thickness}

Figure 17 compares the measured and simulated absorbed doses produced by $28 \mathrm{MeV}$ electrons bombarding the copper targets with 2 and $3 \mathrm{~cm}$ thicknesses at 0 to 120 degrees to the beam axis. The absorbed doses are normalized with the incident beam charge. The MCNPX simulation shows absorbed doses (thick solid line) made up of electrons and photons. The simulated absorbed doses agree well with the experimental results. At 0 degrees, the photon and electron absorbed doses show the same values. On the other hand, from 30 to 120 degrees the electron doses constitute 70 to $80 \%$ of the total absorbed doses. Below $15 \mathrm{~mm}$ in thickness, the electron absorbed doses increase steeply with decreasing target thickness at 0 to 60 degrees because of the primary electrons transmitted through the target. The absorbed doses at 0 degrees are decreased to $29 \%$ in the copper target from 20 to $60 \mathrm{~mm}$ in thickness. However, at wider angles the absorbed doses show constant values because the electron beams stopped at the same depth in the target despite the different target thicknesses. The 
ANGLE ABSORBED-DOSE DISTRIBUTION OF BREMSSTRAHLUNG
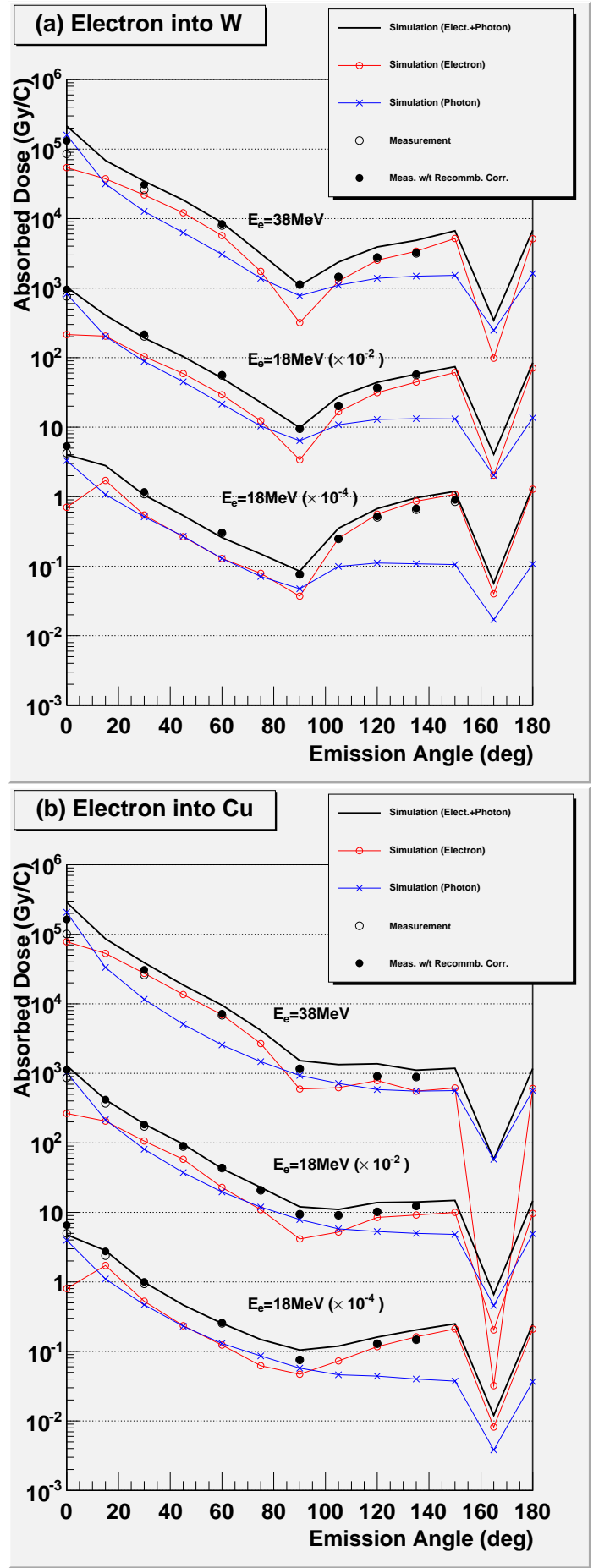

Figure 15. Measured and simulated angular distributions of absorbed doses using PMMA covered ion chamber. Others are the same as in Fig. 14.

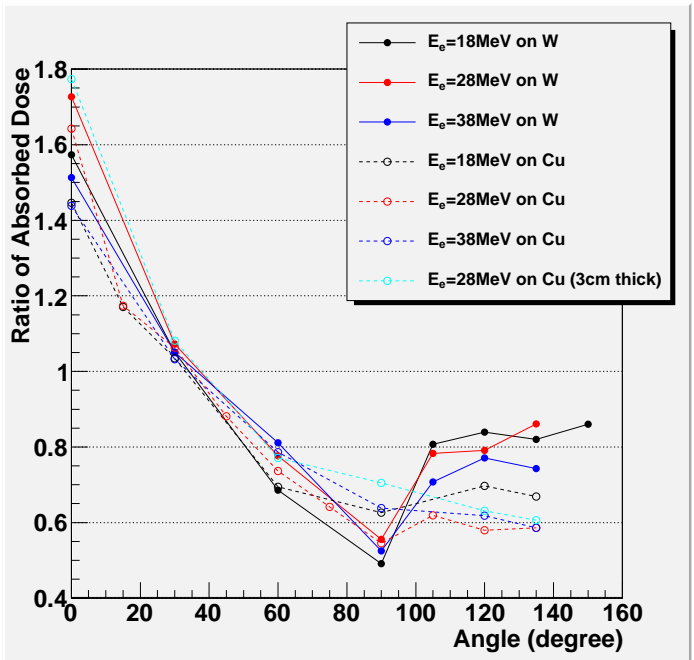

Figure 16. Absorbed-dose ratio of measurement using build-up capped or bare ion chamber.

thickness of the side surface of the target is not changed by increasing the target thickness. Then, electron transport is not affected through the side surface of the target.

\section{Electron Energy}

Absorbed doses were simulated as a function of incident electron energy bombarding the tungsten and copper targets, as shown in Fig. 18. The simulation shows summed absorbed doses of electrons and photons, plotted as thick solid lines. For both targets, the simulation agrees well with the experimental results, shown as filled circles. But at 0 degree the simulated absorbed doses indicate steeper slopes than the measurement with incident electron energy. At forward angles from 0 to 60 degrees, the absorbed doses increase with electron energy due to larger electromagnetic shower events produced by higher energy electrons. Moreover, the photon absorbed doses increase at all angles because electromagnetic shower events are larger when produced by higher energy electrons.

\section{Normalized Angular Distributions}

The measured angular distributions of absorbed doses of $\mathrm{W}, \mathrm{Cu}, \mathrm{Al}$, and $\mathrm{C}$ targets bombarded by 18,28 , and $38 \mathrm{MeV}$ electron beams with the bare ion chamber are normalized at 90 degrees, as shown in Figs. 19-(a) to (c), respectively. The normalized angular distributions at the forward angles decrease with increasing angles; however, the angular distributions at the backward angles depend on the target species. The backward distributions from 90 to 150 degrees indicate different 
M.TAKADA ET AL

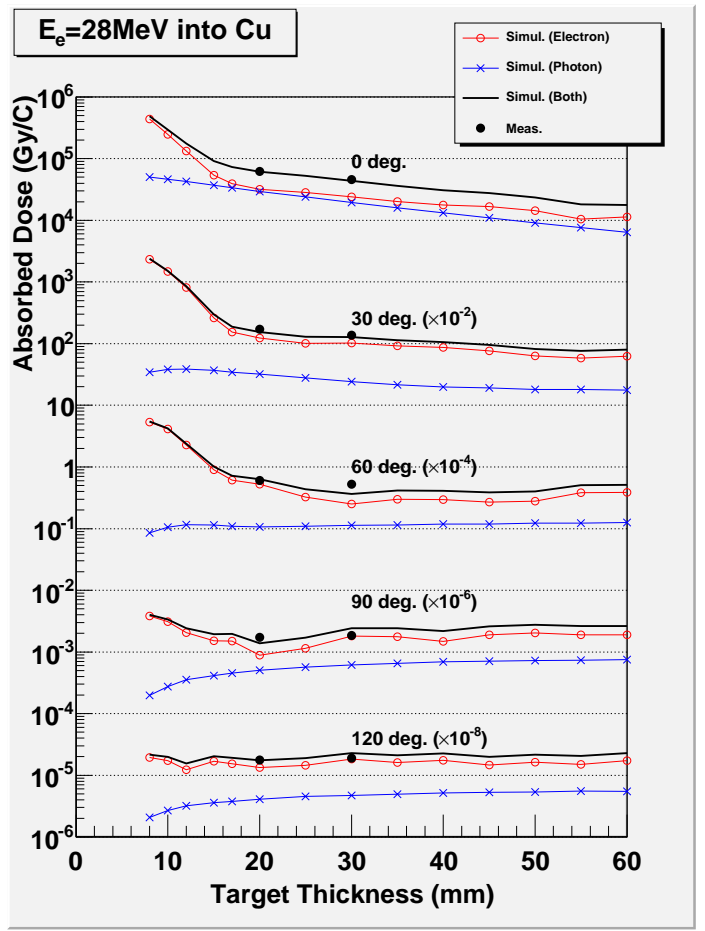

Figure 17. Measured and simulated absorbed-dose distributions with target thicknesses produced by $28 \mathrm{MeV}$ electrons bombarding $\mathrm{Cu}$ target at 0 to 120 degrees with respect to the electron beam axis. Black filled circles show the measured absorbed doses. Thick solid line shows the simulated absorbed doses summed of electrons (solid line with open circle) and photons (solid line with cross mark).

shapes than those at the forward angles. At the backward angles, targets with larger atomic numbers show larger absorbed doses because backscattered electron flux is proportional to the atomic number [15].

A large target (carbon) shows constant absorbed doses at backward angles, from 100 to 150 degrees. The carbon target is thick enough to stop secondary electrons escaping from the side surface of the target.

\section{Attenuation Profile}

The attenuation profile of absorbed doses behind PMMA from 0 to $20 \mathrm{~cm}$ in thickness were measured for the 2-cm-thick copper target bombarded by 18 and $28 \mathrm{MeV}$ electrons and for the tungsten target bombarded by $38 \mathrm{MeV}$ electrons, at angles of 0,30 , and 135 degrees to the electron beam axis. In the dose measurement, the ion chamber was covered with the PMMA build-up cap. Figure 20 shows the attenuation curves of experimental absorbed doses, shown as filled circles. The measured results are compared with the simulation of summed absorbed doses, shown as thin solid
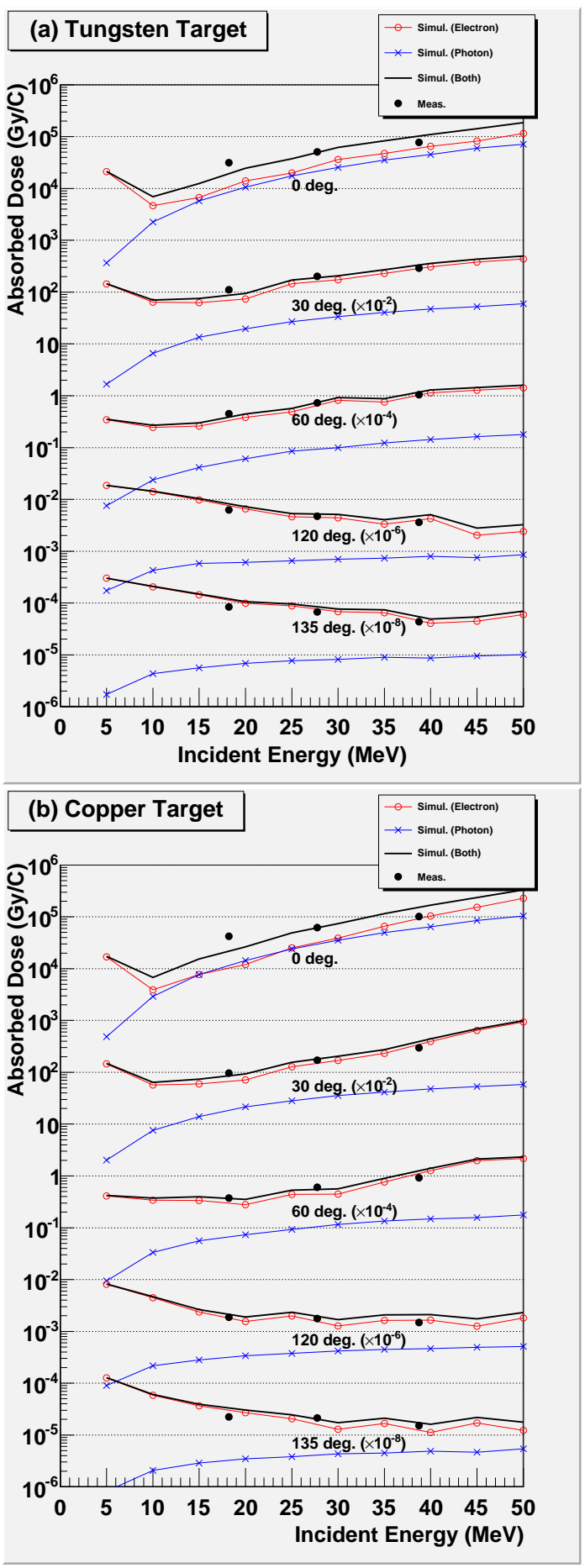

Figure 18. Measured and simulated absorbed-dose distributions at 0 to 135 degrees as a function of incident electron energy bombarding (a) tungsten and (b) copper targets. The others are the same as in Fig. 17. 

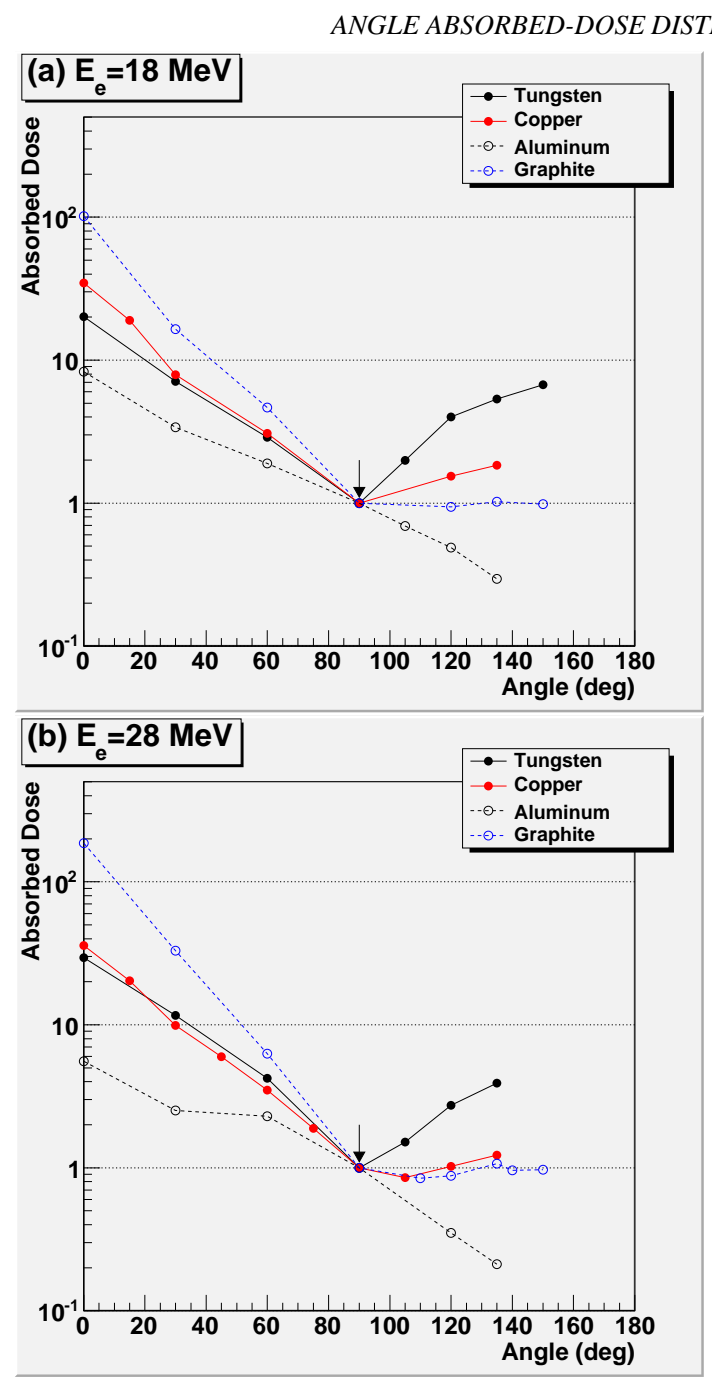

Figure 19. Measured angular distributions of absorbed dose, normalized at 90 degree: (a) 18, (b) 28, and (c) $38 \mathrm{MeV}$ electron beams.

lines, of photons (thin solid lines with cross marks) and electrons (thin lines with open circles). The simulation shows smaller values than the measurement $(\mathrm{C} / \mathrm{E}=0.33$ - 0.85). These smaller simulated values may be attributable to the angular dose distributions of secondary particles produced by the target. The photons with a sharp peak at 0 degrees are incident on the PMMA plates in the measurements. On the other hand, in the simulation, the photon and electron energy spectra with flat angular distributions are incident on the PMMA plates. The simulated absorbed doses are multiplied by factors, described as numerical values in square brackets, for comparison with the measured results, shown as thick solid lines.

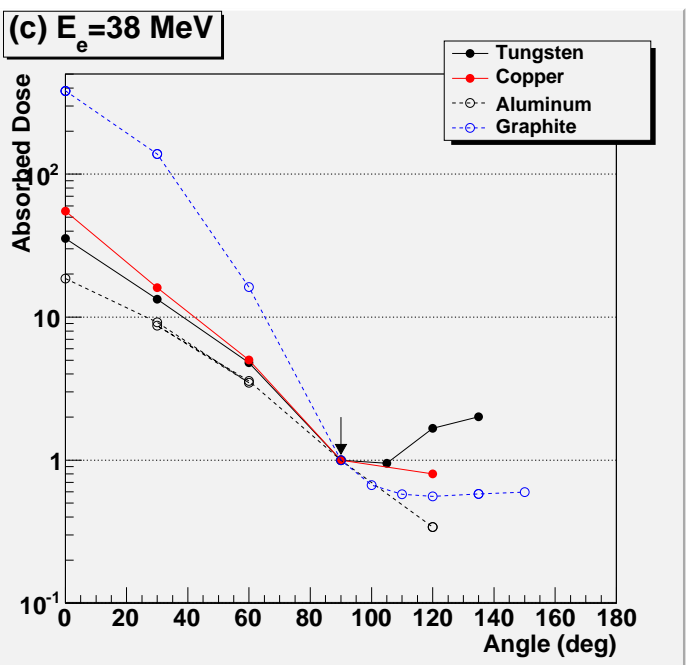

Figure 19. Continued

The experimental attenuation curves of absorbed doses agree well with the simulated results in relative values. The attenuation curves of absorbed doses are the sums of the electron and photon doses. In the attenuated absorbed doses at shallow depths, absorbed doses consist primarily of electrons. However, at thicker plates, the contribution of electrons is negligible and the absorbed doses are mainly from photons. The measured attenuation curve of a copper target bombarded by 18 $\mathrm{MeV}$ electron beams at 0 degrees indicates a broad peak from 0 to $5 \mathrm{~cm}$ in depth. However, the simulation shows a smaller broad peak. No broad peak is observed in the other targets and energies. The measured attenuation curve, except for the aluminum target bombarded by 18 $\mathrm{MeV}$ electrons at 0 degrees, is similar to the simulated attenuation profiles from 0 to $20 \mathrm{~cm}$ in thickness.

\section{CONCLUSION}

The angular distributions of absorbed doses of Bremsstrahlung photons and secondary electrons across a wide range of emission angles were experimentally obtained, using bare or PMMA build-up capped ion-chamber with $0.6 \mathrm{~cm}^{3}$ air volume. The Bremsstrahlung photons and electrons were produced by 18,28 , and $38 \mathrm{MeV}$ electron beams bombarding the tungsten, copper, aluminum, and carbon targets. The absorbed doses were obtained from simulated photon and electron energy spectra by multiplying the response functions of ion chambers for photons and electrons up to $50 \mathrm{MeV}$, simulated with the MCNPX code. The simulated angular distributions of absorbed doses agree with the experimental results, within a factor of 1.8 for $\mathrm{W}, \mathrm{Cu}$, and $\mathrm{Al}$ targets, and 
M.TAKADA ET AL
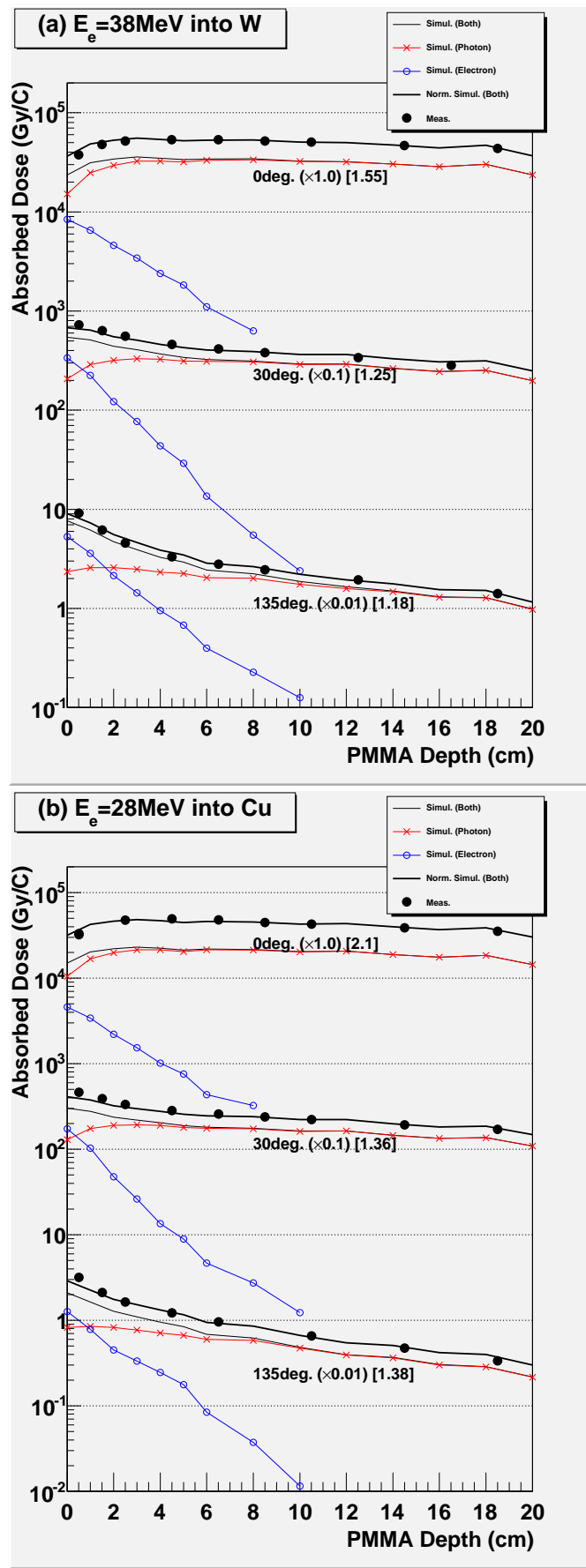

Figure 20. Measured and simulated absorbed-dose distributions behind PMMA plates, produced by $38 \mathrm{MeV}$ electrons in tungsten target at 0,30 , and 135 degrees. The absorbed doses were measured using build-up capped ion-chamber and plotted as filled circles. Thin solid line shows the simulated absorbed doses summed of electrons (solid line with open circle) and photons (solid line with cross mark).

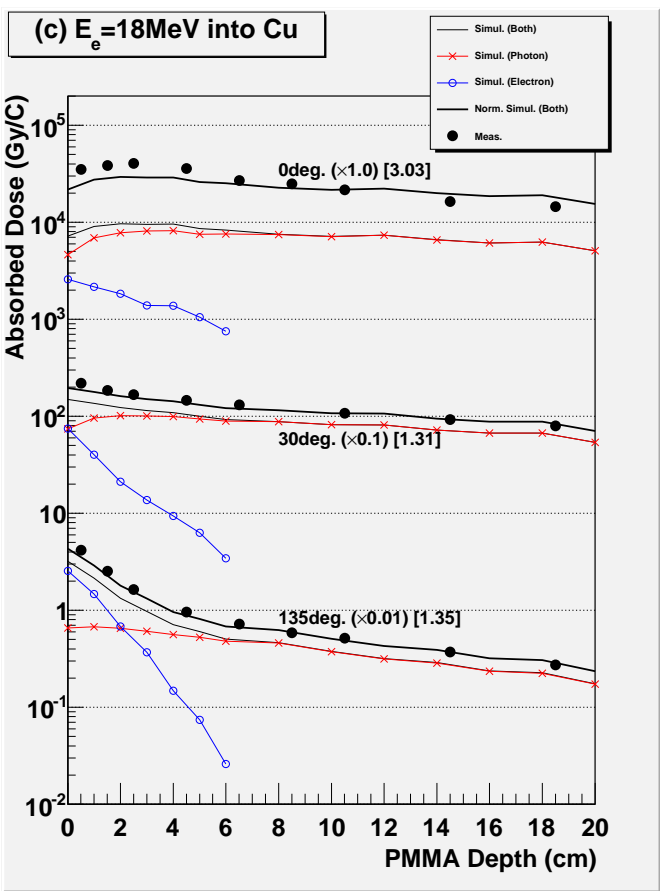

Figure 20. Continued

within a factor of 2 for the $\mathrm{C}$ target across wide ranges of emission angles, incident electron energies, and atomic numbers of targets.

The dependences of absorbed doses on electron energy and target thickness are compared between the measurement and simulation, produced by tens of $\mathrm{MeV}$ electron beams bombarding several targets. The simulated results agree well with the experimental results. The measured attenuation profiles of absorbed doses agree with the experimental results in relative value at several incident electron energies and at over 30 degrees. The experimental angular distributions at the forward angle are similar to each other at several targets and electron energies. On the other hand, the absorbed doses at the backward angles depend on the target species. The backscattered absorbed doses are found to be important for evaluating electron absorbed doses around the Bremsstrahlung target.

Our angular absorbed-dose distributions of Bremsstrahlung photons and secondary electrons are useful not only to estimate doses for patients, but also to optimize the shielding of the linear accelerator room.

\section{REFERENCES}

1. Japan Radioisotope Association, "Statistics on the use of radiation in Japan,” (in Japanese), 
http://www.jrias.or.jp/index.cfm/6,1246,107,132,html,13. B.A. Faddegon, C.K.Ross, and D.W.O.Rogers, (access 2011/12).

2. Marco Silari, "Applications of Particle Accelerators in Medicine," Radiat. Protect. Dosim. 146, 440-450 (2011).

3. MCNPX User's Manual, Los Alamos Natl. Lab. rep., LA-CP-05-0369 (2005). Info. on MCNPX at URL http://mcnpx.lanl.gov/. (access 2011/12).

4. M.J.Berger, J.S.Coursey, M.A.Zucker, and J.Chang, "Stopping-Power and Range Tables for Electrons, Protons, and Helium Ions,"

http://www.nist.gov/pml/data/star/index.cfm (access 2011/12).

5. R.E.Ellis, L.R.Read, "Recombination in Ionization Chambers Irradiated with Pulsed Electron Beams I: Plane Parallel Plate Chamber," Phys. Med. Biol. 14[2], 293-304 (1969).

6. J.W.Boag and J.Currant, "Current Collection and ionic recombination in small cylindrical ionization chambers exposed to pulsed radiation," Br. J. Radiol. 53, 471-478 (1980).

7. K.Kosako, K.Oishi, T.Nakamura, M.Takada, K.Sato, T.Kamiyama, and Y.Kiyanagi, "Angular Distribution of Photoneutrons from Copper and Tungsten Targets Bombarded by 18, 28 and $38 \mathrm{MeV}$ Electrons," J. Nucl. Scien. Techn. 47[3], 286-294 (2011).

8. Y.Watanabe, T.Fukahori, H.Nakashima, S.Chiba, K.Kosako, N.Shigyo, T.Murata, N.Yamano, T.Hino, and K.Maki, "Nuclear Data Evaluations for JENDL High-Energy File," Proc. Int. Conf. Nucl. Data for Sci. and Technol., Santa Fe, NM (United States), 26 Sep - 1 Oct 2004, 769[1] (2005).

9. M.B.Chadwick, P.G.Young, S.Chiba, S.C.Frankle, G.M.Hale, H.G.Hughes, A.J.Koning, R.C.Little, R.E.MacFarlane, R.E.Prael, and L.S.Waters, "CrossSection Evaluations to $150 \mathrm{MeV}$ for AcceleratorDriven Systems and Implementation in MCNPX," Nucl. Sci. Eng. 131[3], 293-328 (1999).

10. K.J.Adams, "Electron Upgrade for MCNP4B," Los Alamos Natl. Lab. Intern. Memo. X-5-RN(U)-0014, (2000).

11. M.C.White, "Photoatomic Data Library MCPLIB04: A New Photoatomic Library Based on Data from ENDF/V-VI Release 8," Los Alamos Natl. Lab. Intern. Memo. X-5-MCW-02-111, (2002).

12. N.Reynaert, H.Palmans, H.Thierens, and R.Jeraj, "Parameter dependence of the MCNP electron transport in determining dose distributions," Med. Phys. 29(10), 2446 - 2454 (2002). "Angular distribution of bremsstrahlung from 15$\mathrm{MeV}$ electrons incident on thick targets of $\mathrm{Be}, \mathrm{Al}$, and Pb," Med. Phys. 18, 727-739 (1991).

14. Y.Kirihara, Y.Namito, H.Iwase, and H.Hirayama, "Monte Carlo simulation of Tabata's electron backscattering experiments," Nucl. Instr. Methods. B 268, 2384-2390 (2010).

15. G.F.Knoll. Radiation Detection and Measurement, 3rd ed. (John Wiley \& Sons, Inc., New York) (1999). 


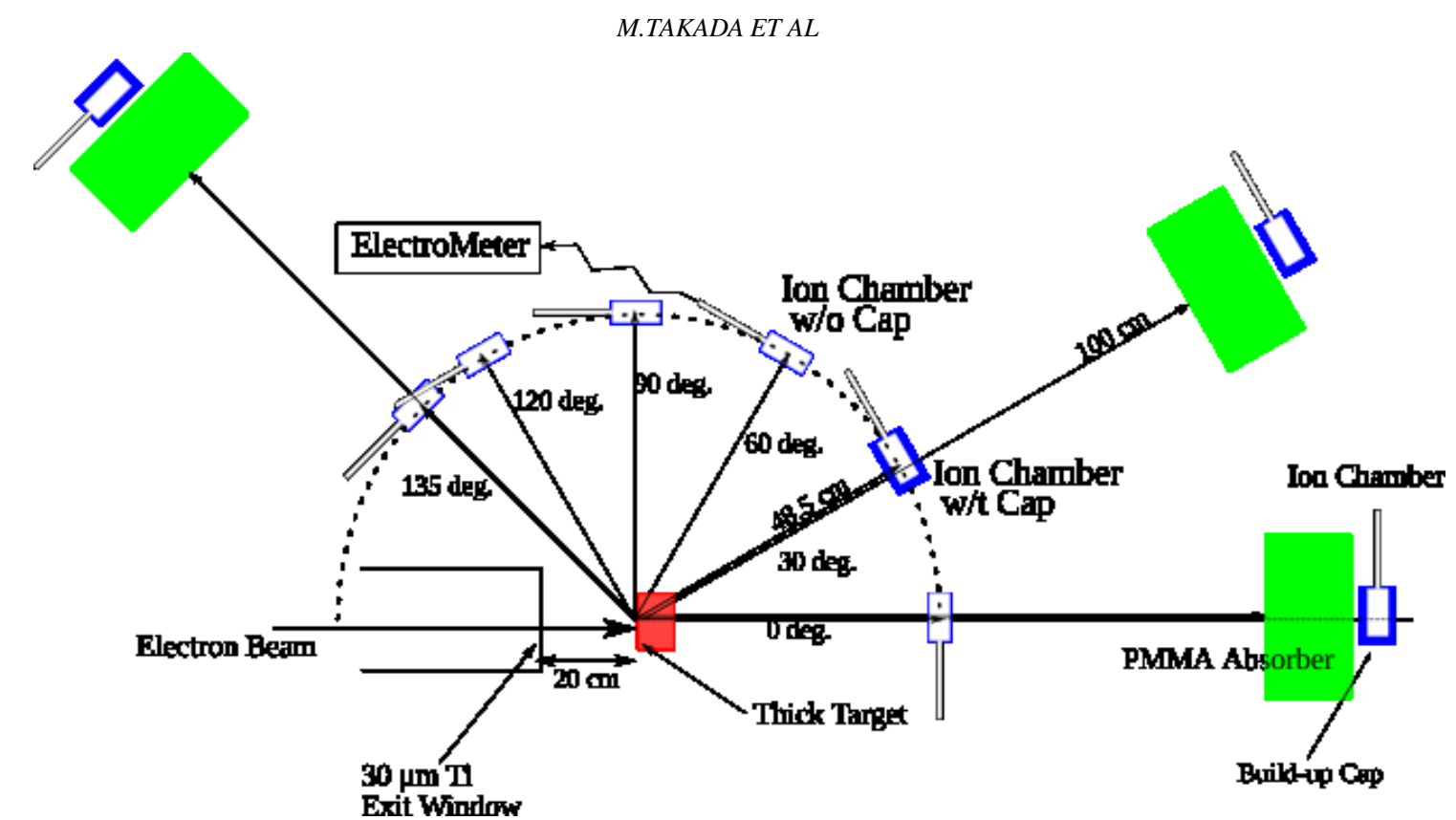

Figure 1. 
ANGLE ABSORBED-DOSE DISTRIBUTION OF BREMSSTRAHLUNG

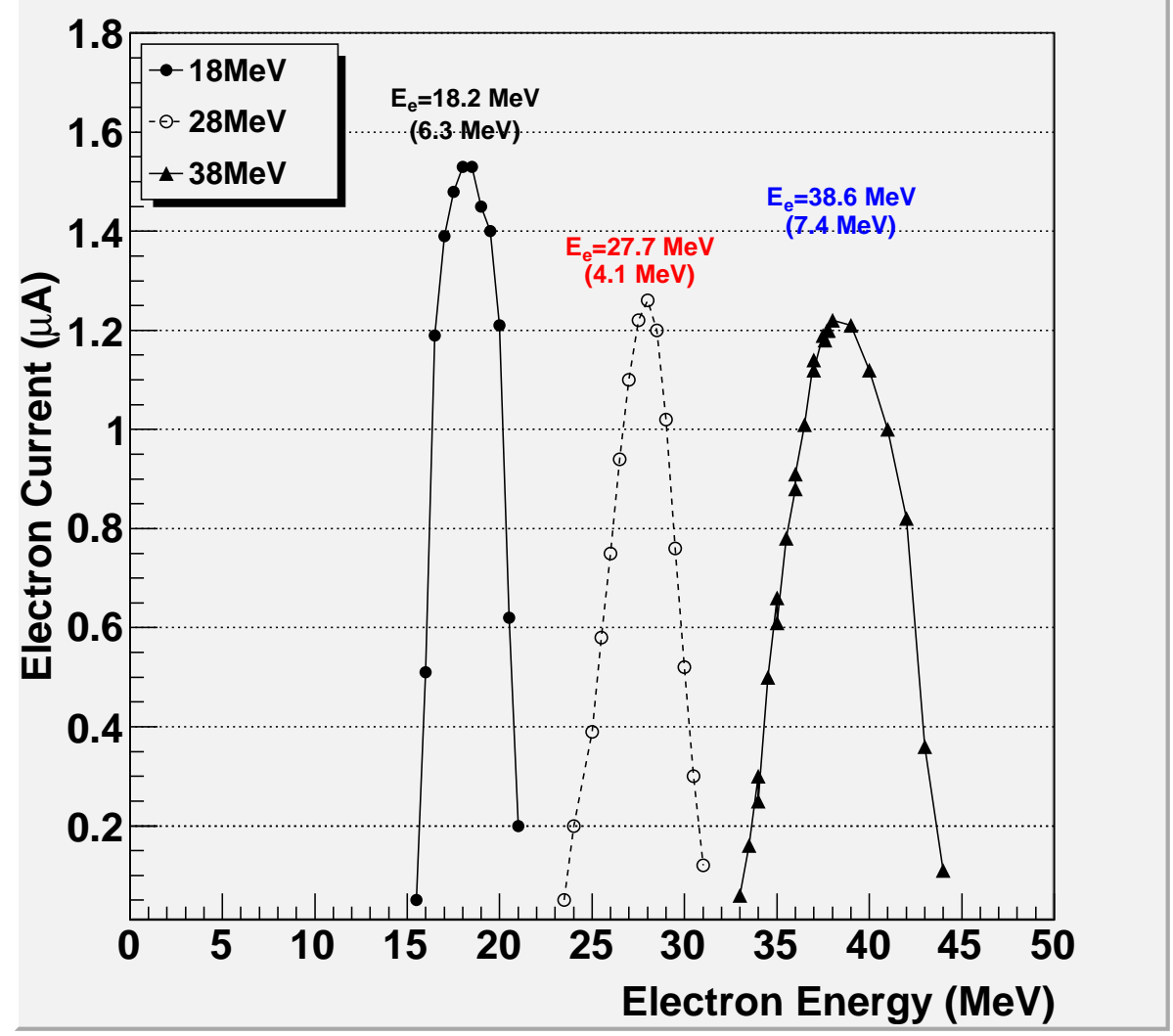

Figure 2. 
M.TAKADA ET AL

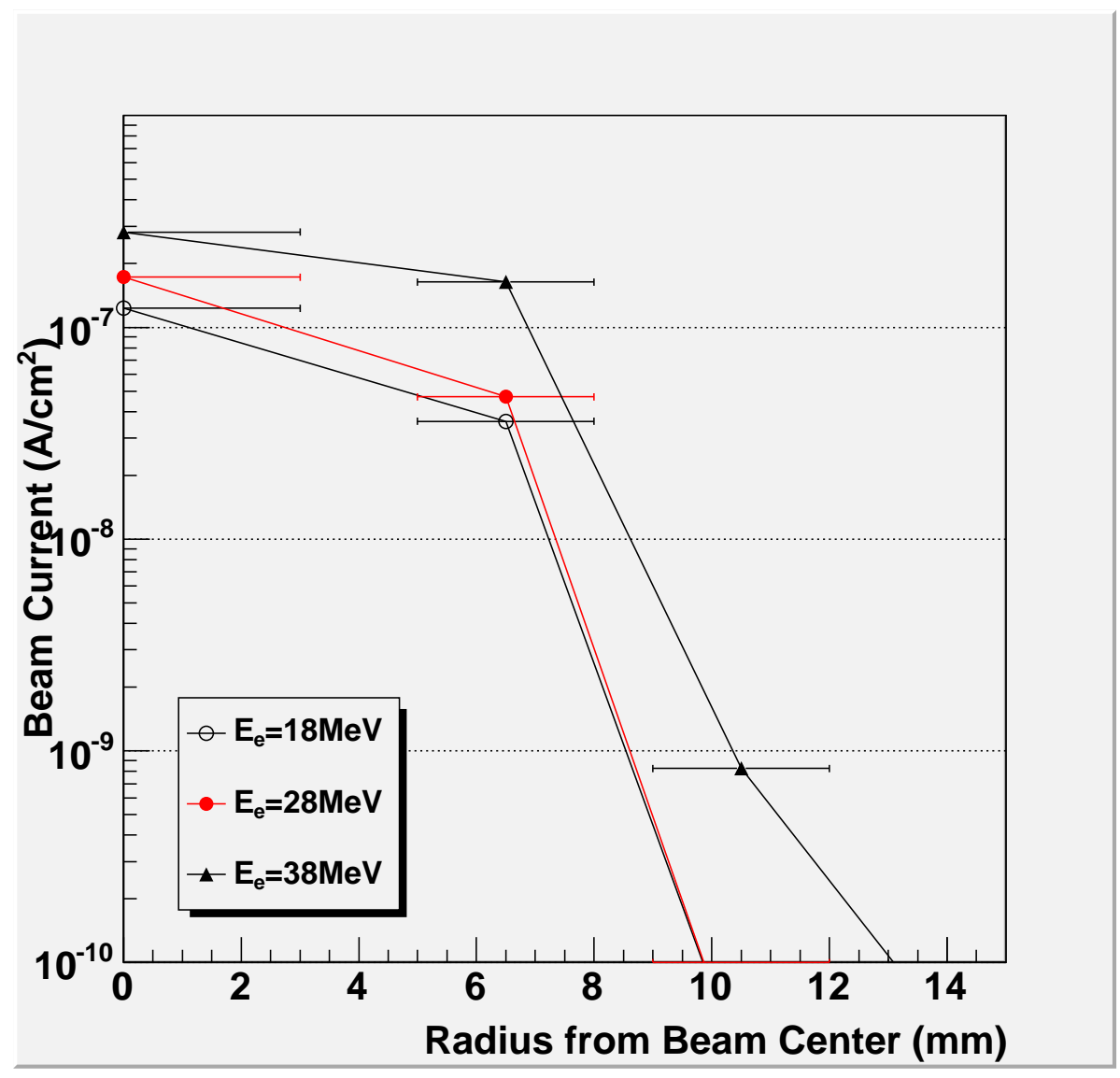

Figure 3. 


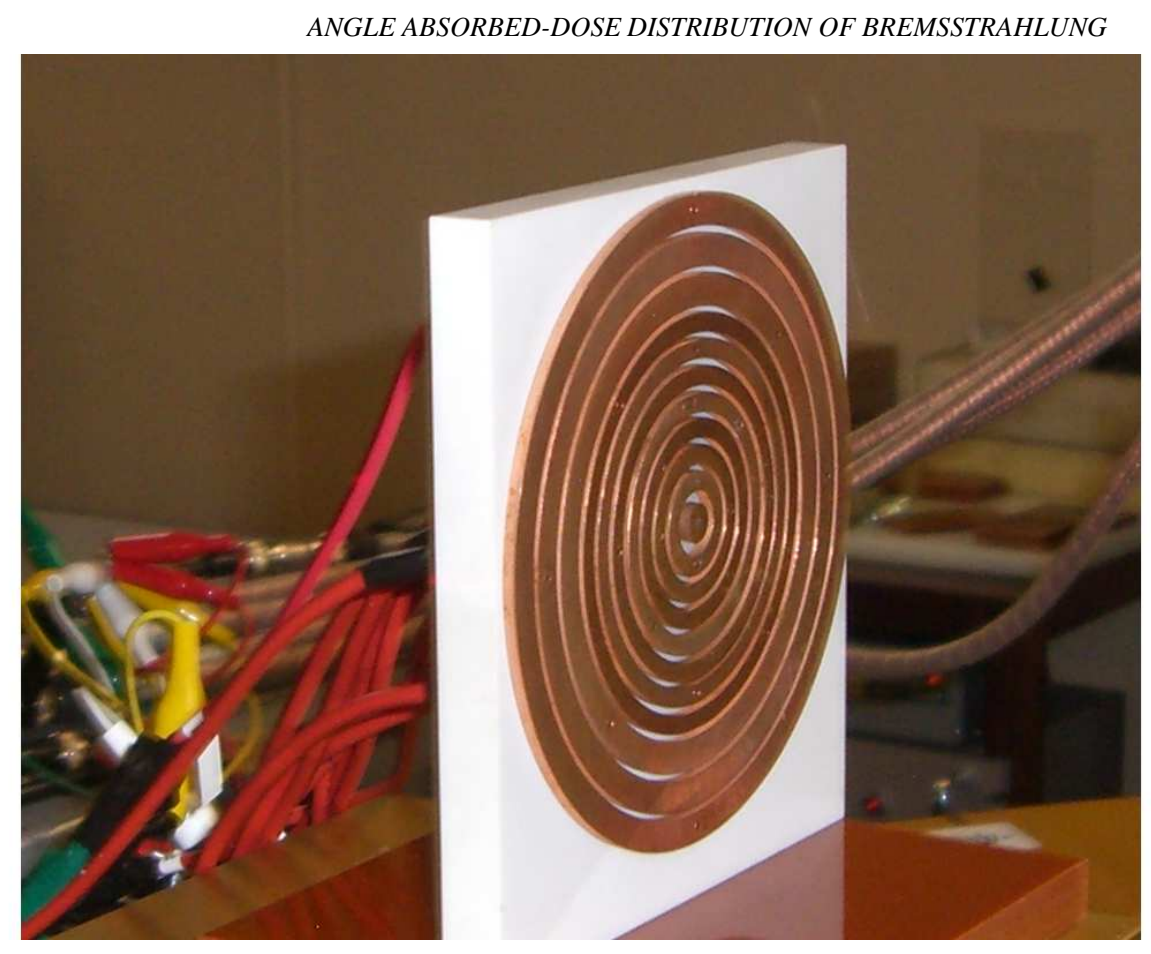

Figure 4 . 
M.TAKADA ET AL

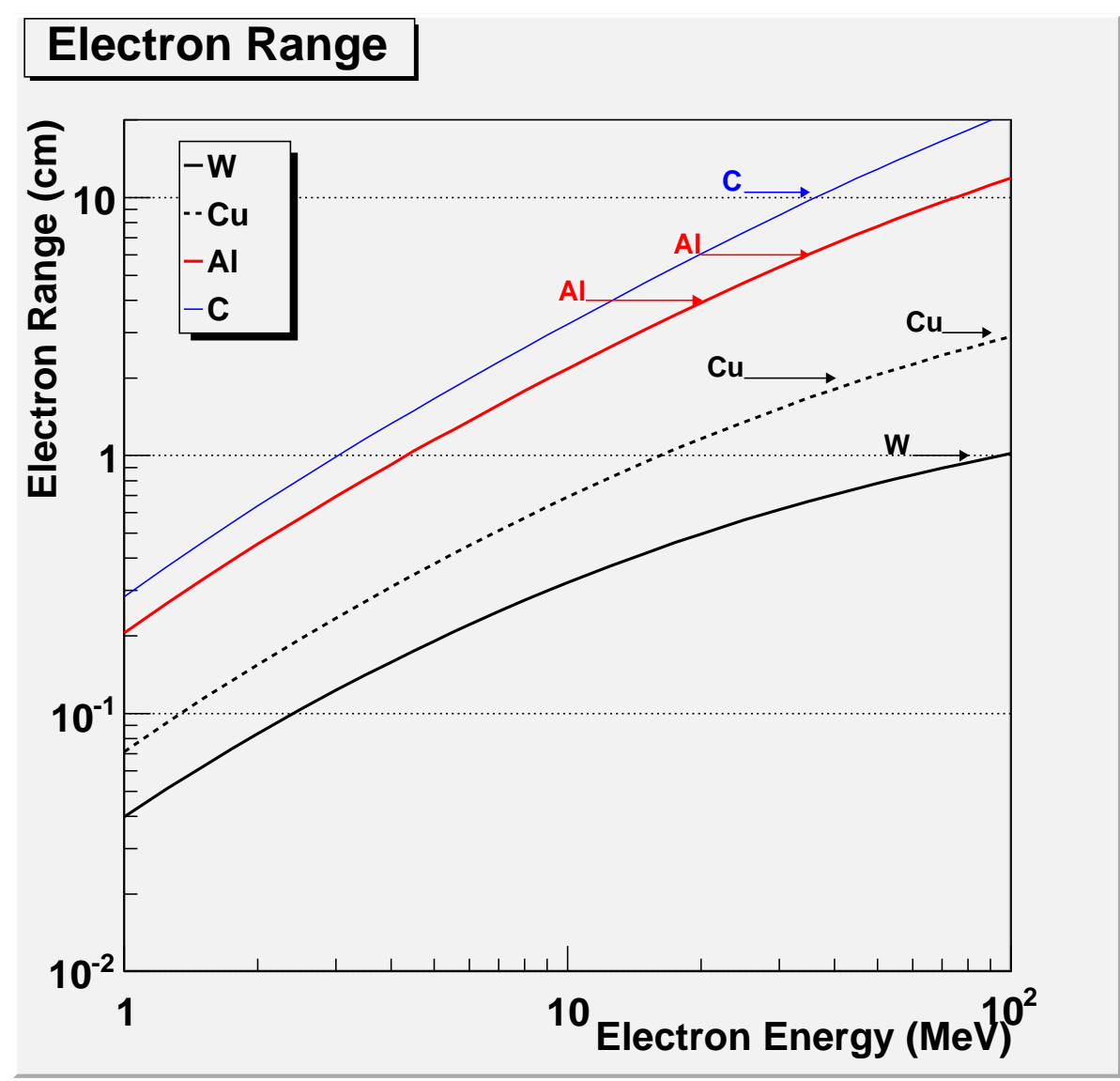

Figure 5. 
ANGLE ABSORBED-DOSE DISTRIBUTION OF BREMSSTRAHLUNG

(a) Ion Chamber

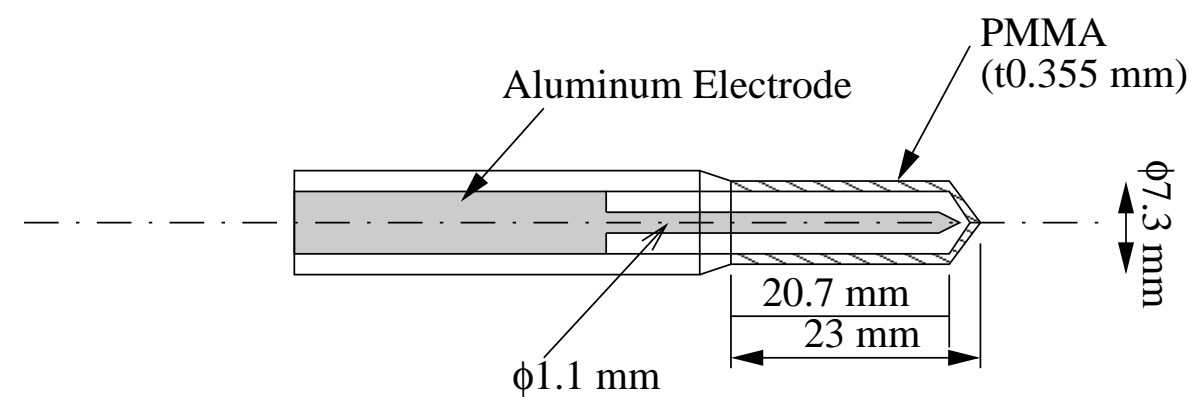

(b) PMMA Build-up Cap

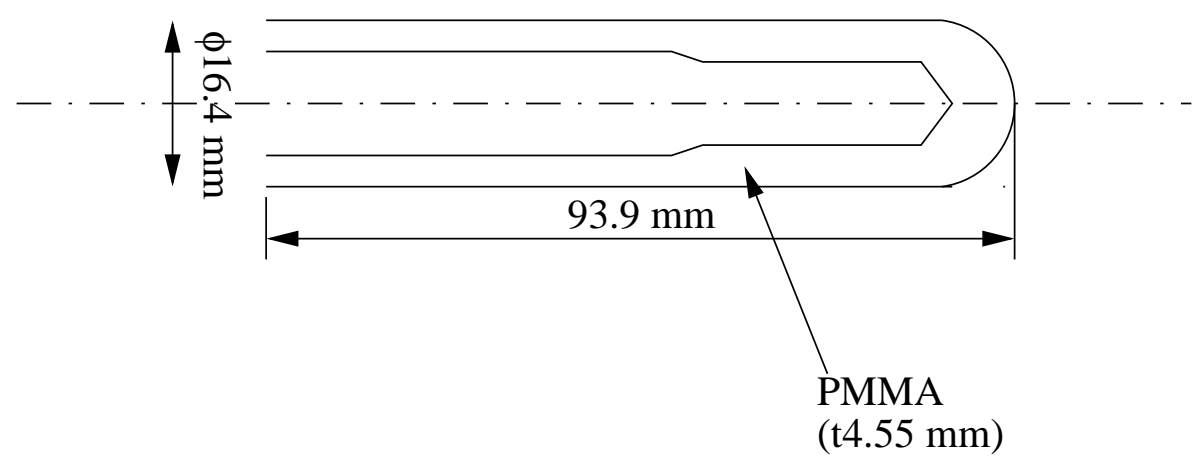

Figure 6.

(a) Bare Ion Chamber

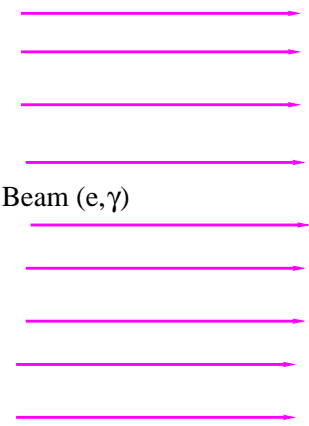

(b) PMMA Caped Ion Chamber

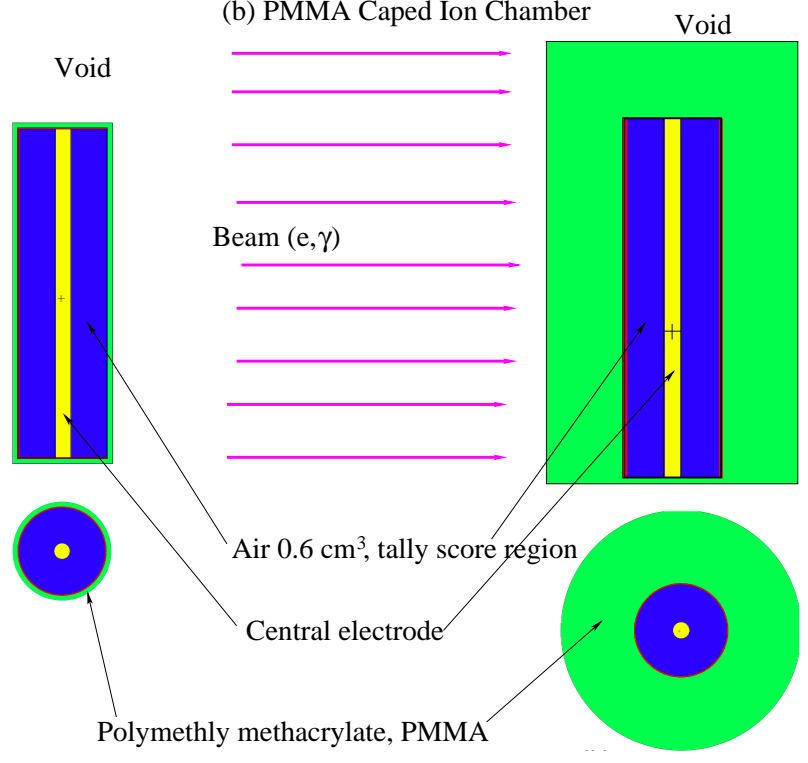

Figure 7. 
M.TAKADA ET AL

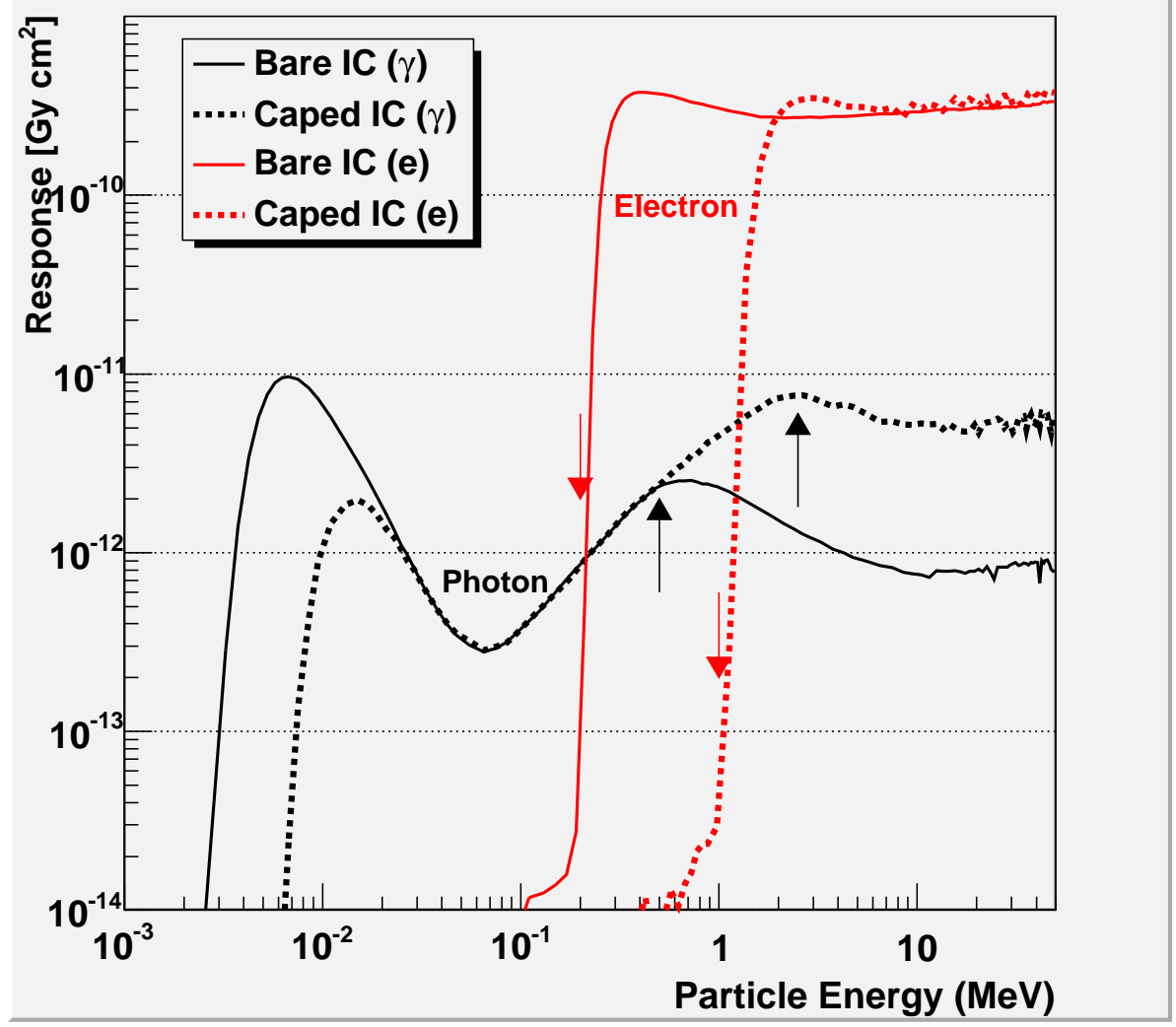

Figure 8. 
ANGLE ABSORBED-DOSE DISTRIBUTION OF BREMSSTRAHLUNG

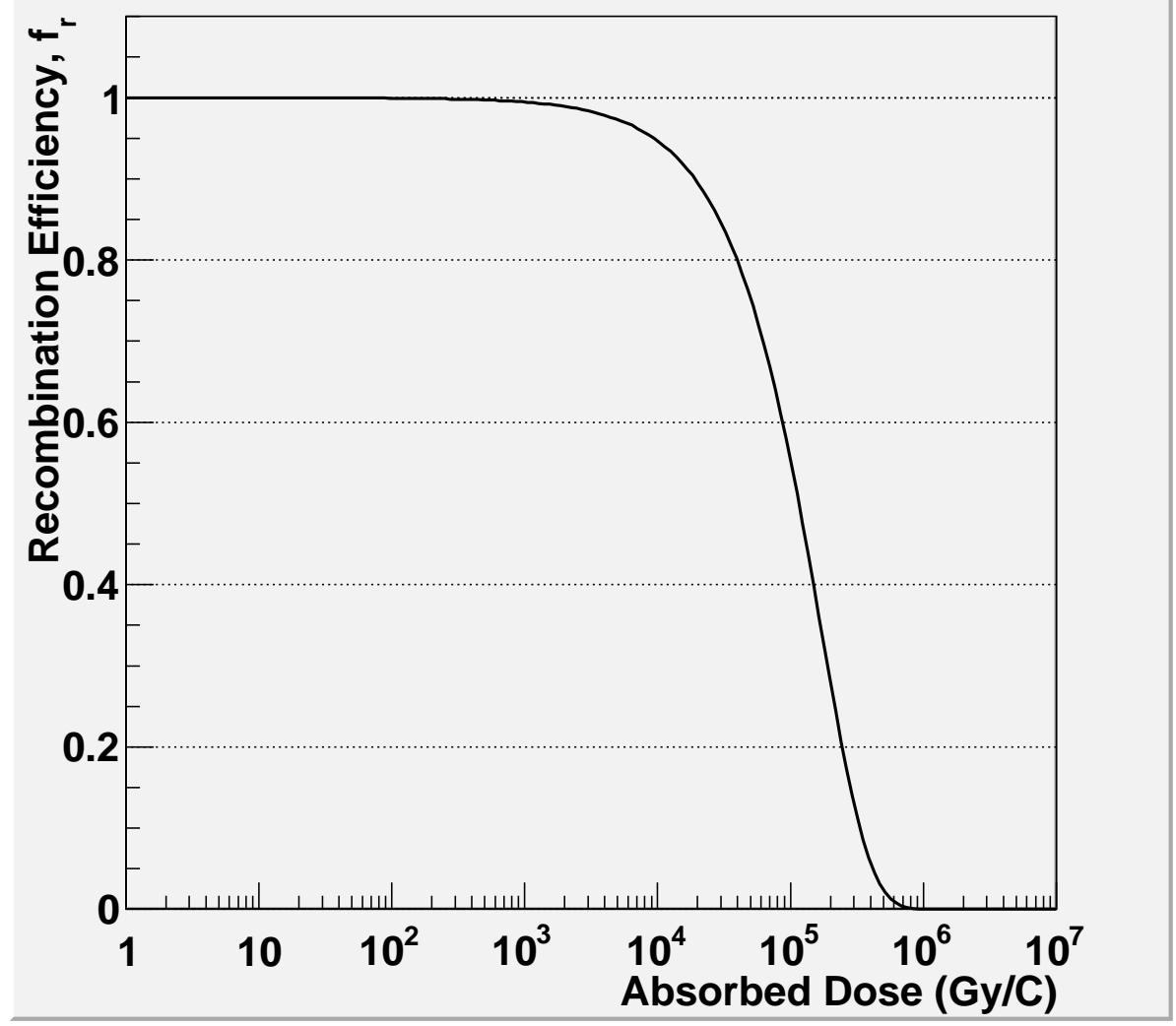

Figure 9. 


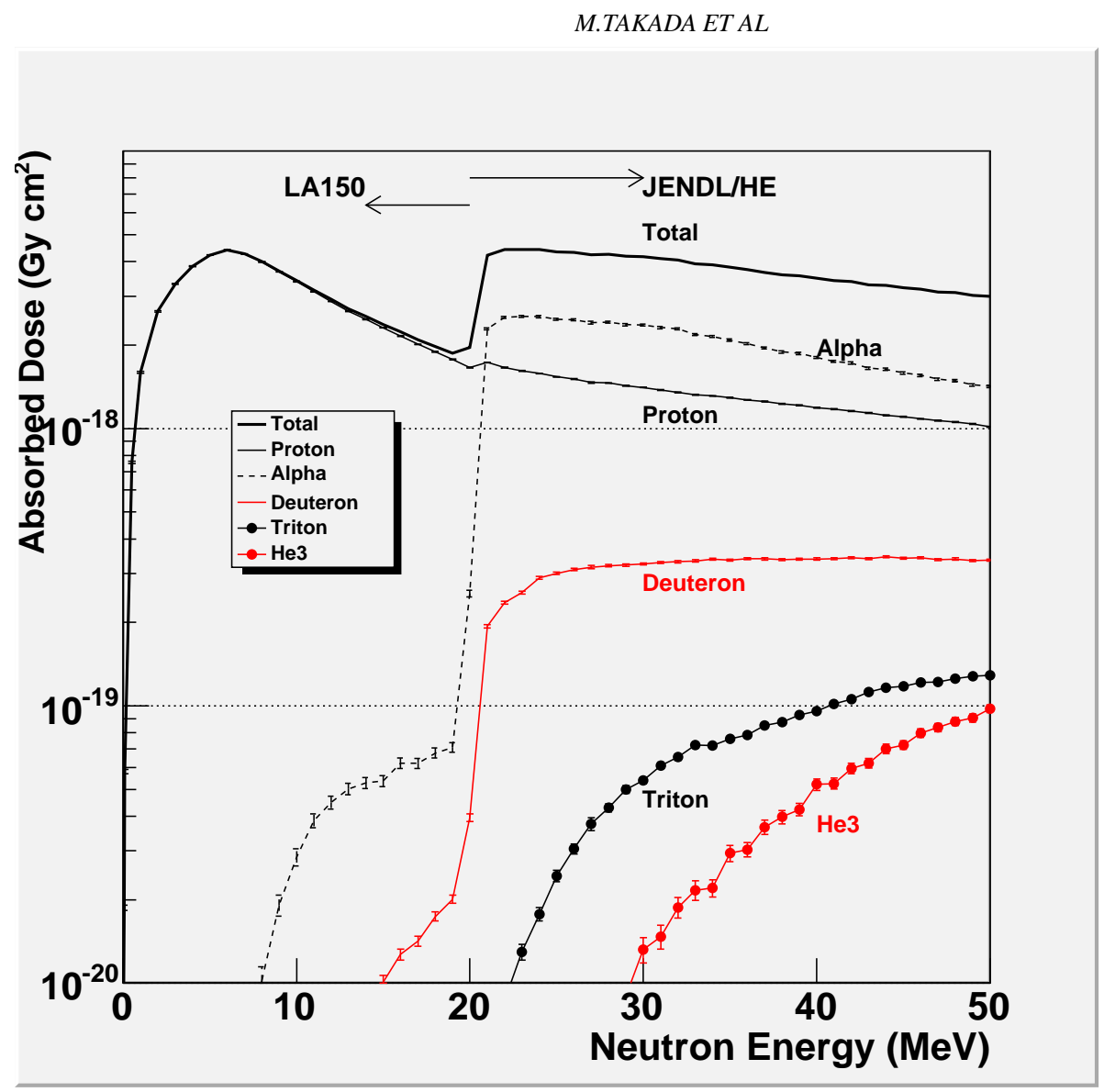

Figure 10. 
ANGLE ABSORBED-DOSE DISTRIBUTION OF BREMSSTRAHLUNG

(a) $E_{e}=15 \mathrm{MeV}$ in $\mathrm{Be}$

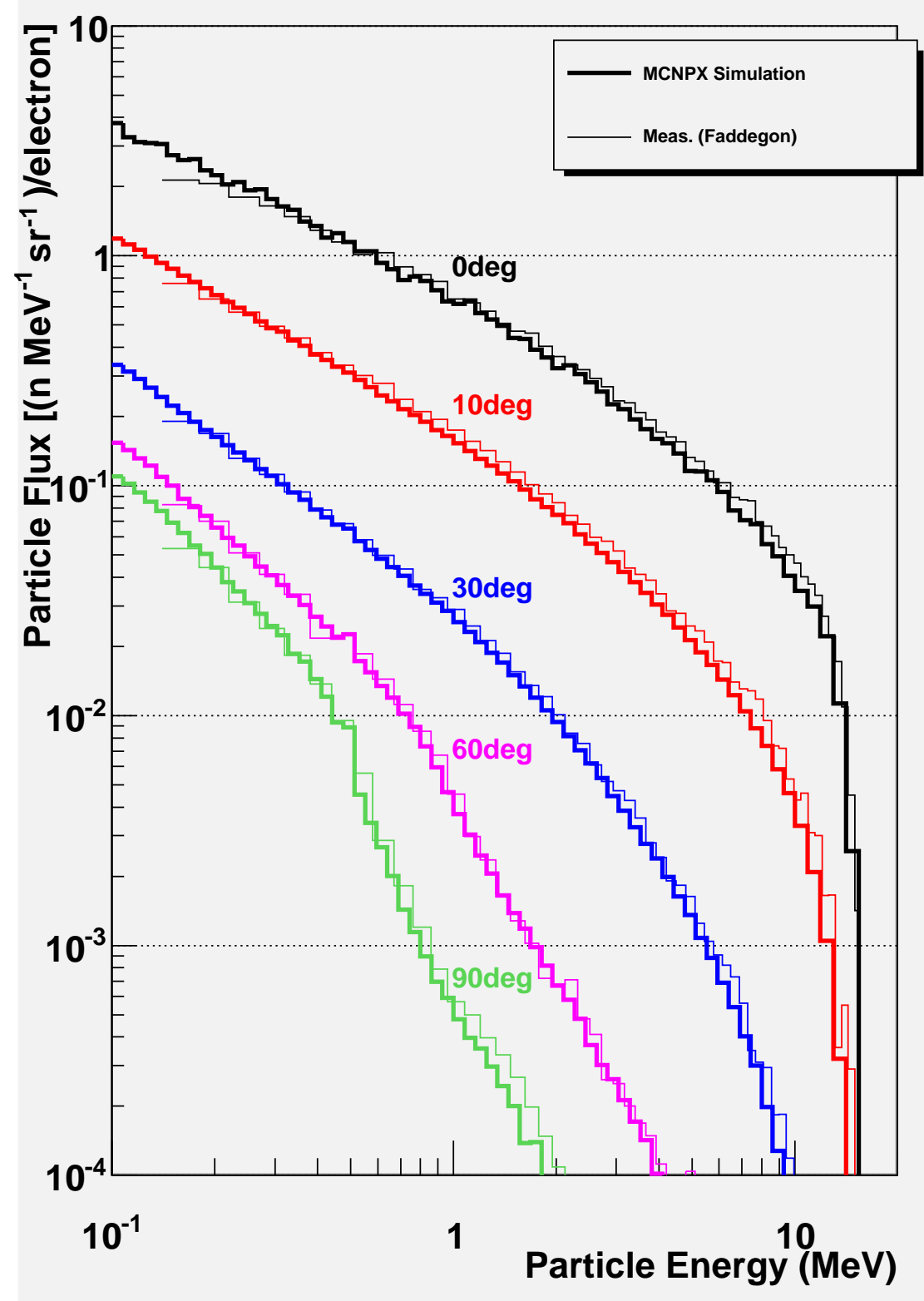

Figure 11 


\section{(b) $E_{e}=15 \mathrm{MeV}$ in $\mathrm{Al}$}

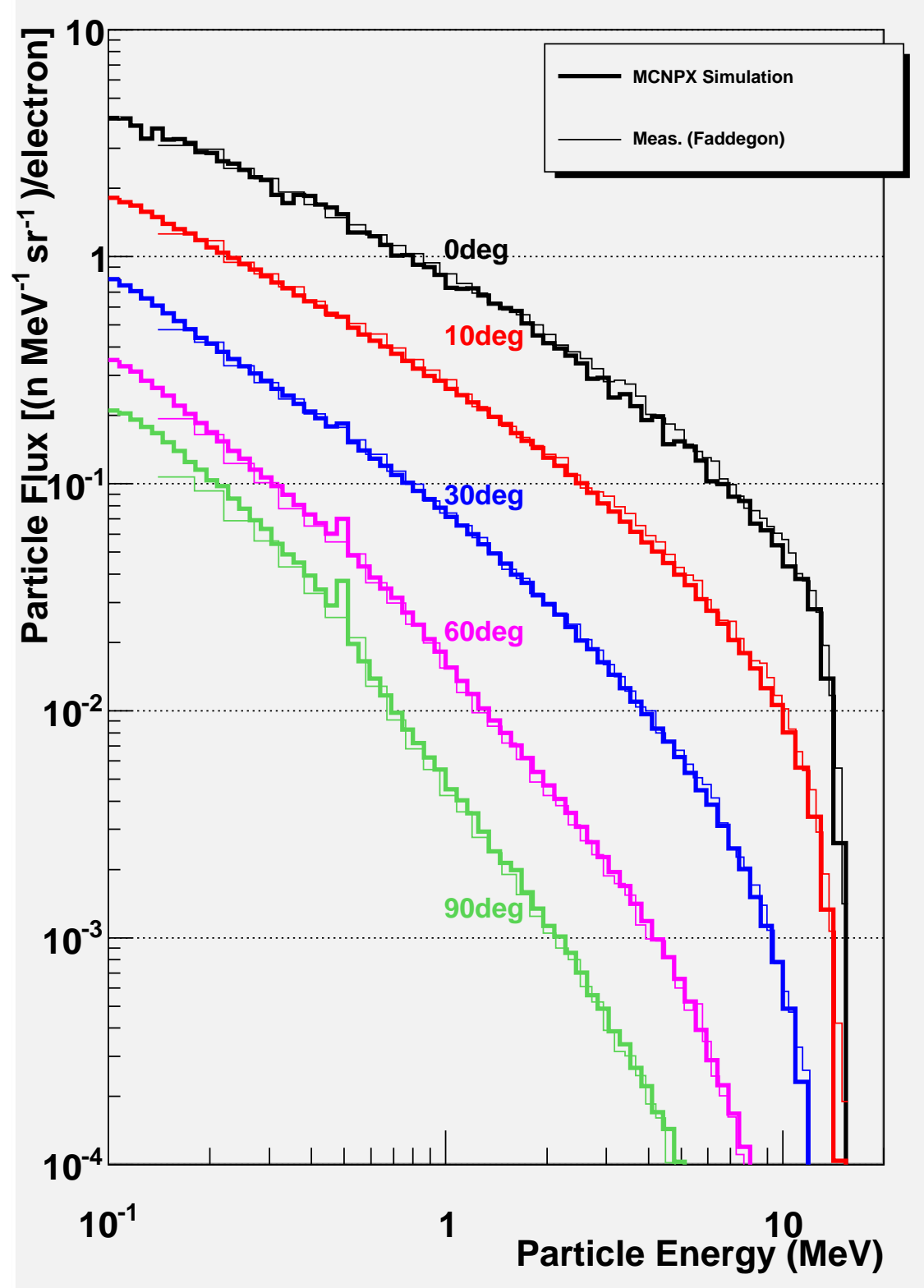

Figure 11 
ANGLE ABSORBED-DOSE DISTRIBUTION OF BREMSSTRAHLUNG
(c) $\mathrm{E}_{\mathrm{e}}=15 \mathrm{MeV}$ in $\mathrm{Pb}$

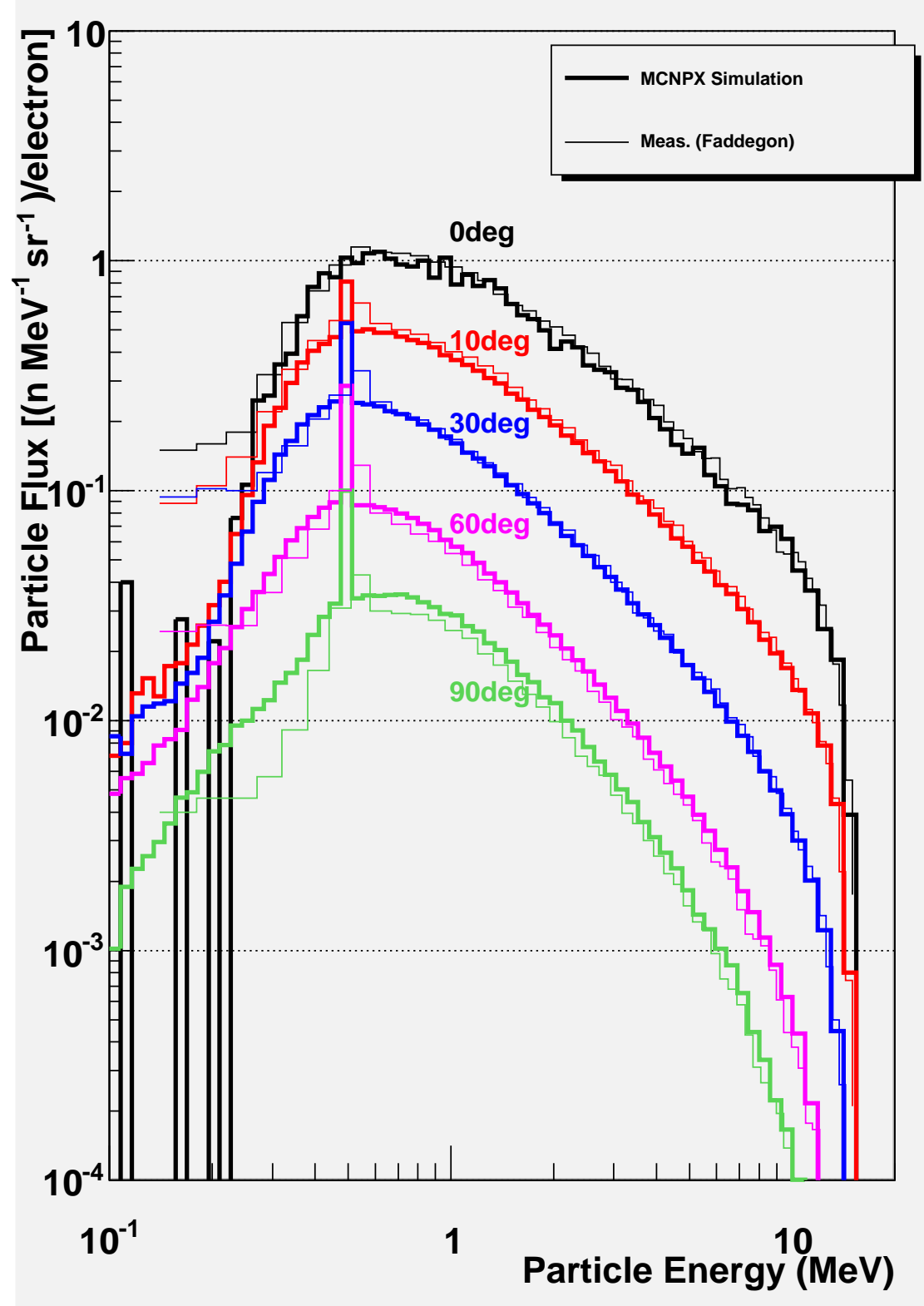

Figure 11. 


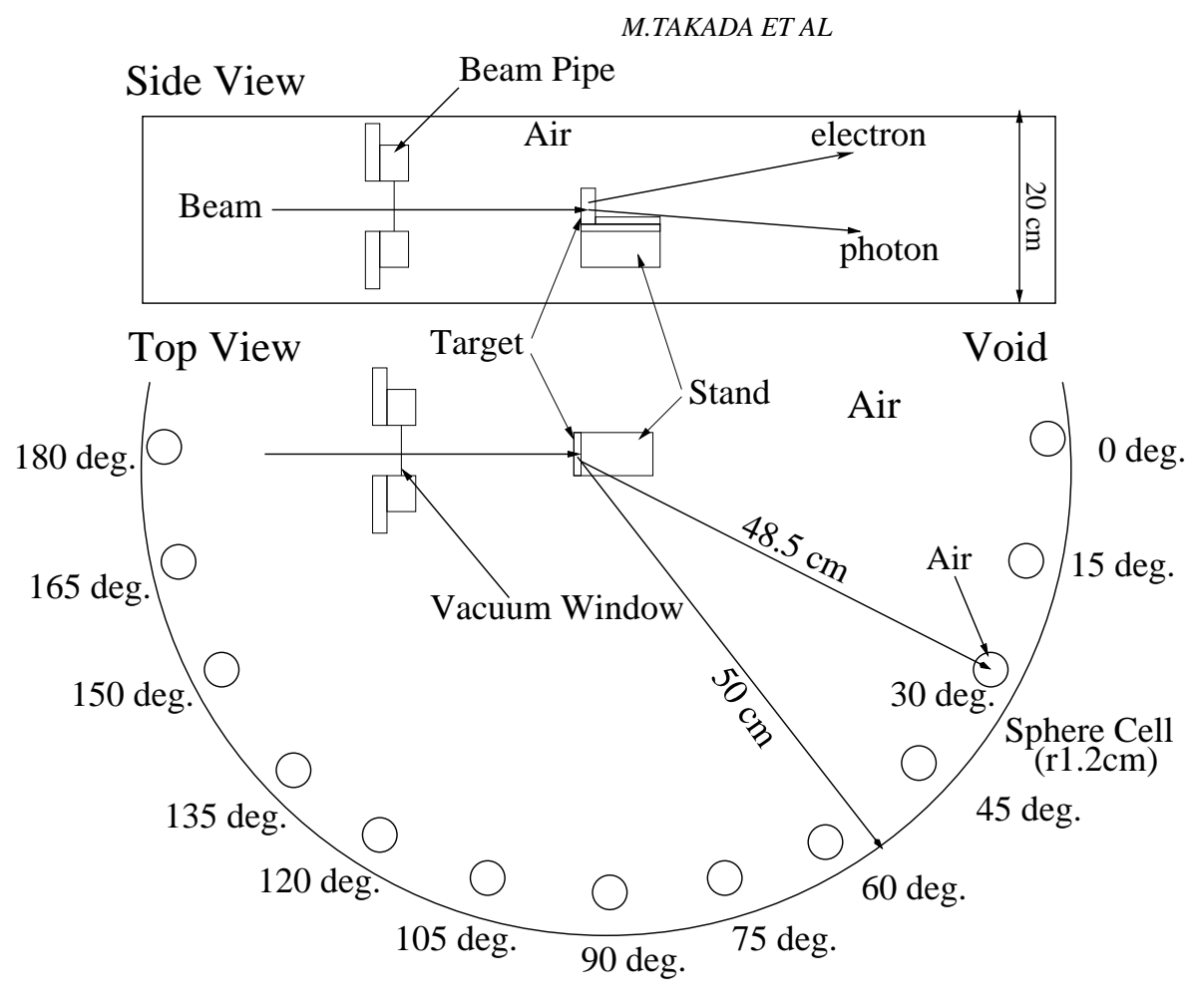

Figure 12. 
ANGLE ABSORBED-DOSE DISTRIBUTION OF BREMSSTRAHLUNG

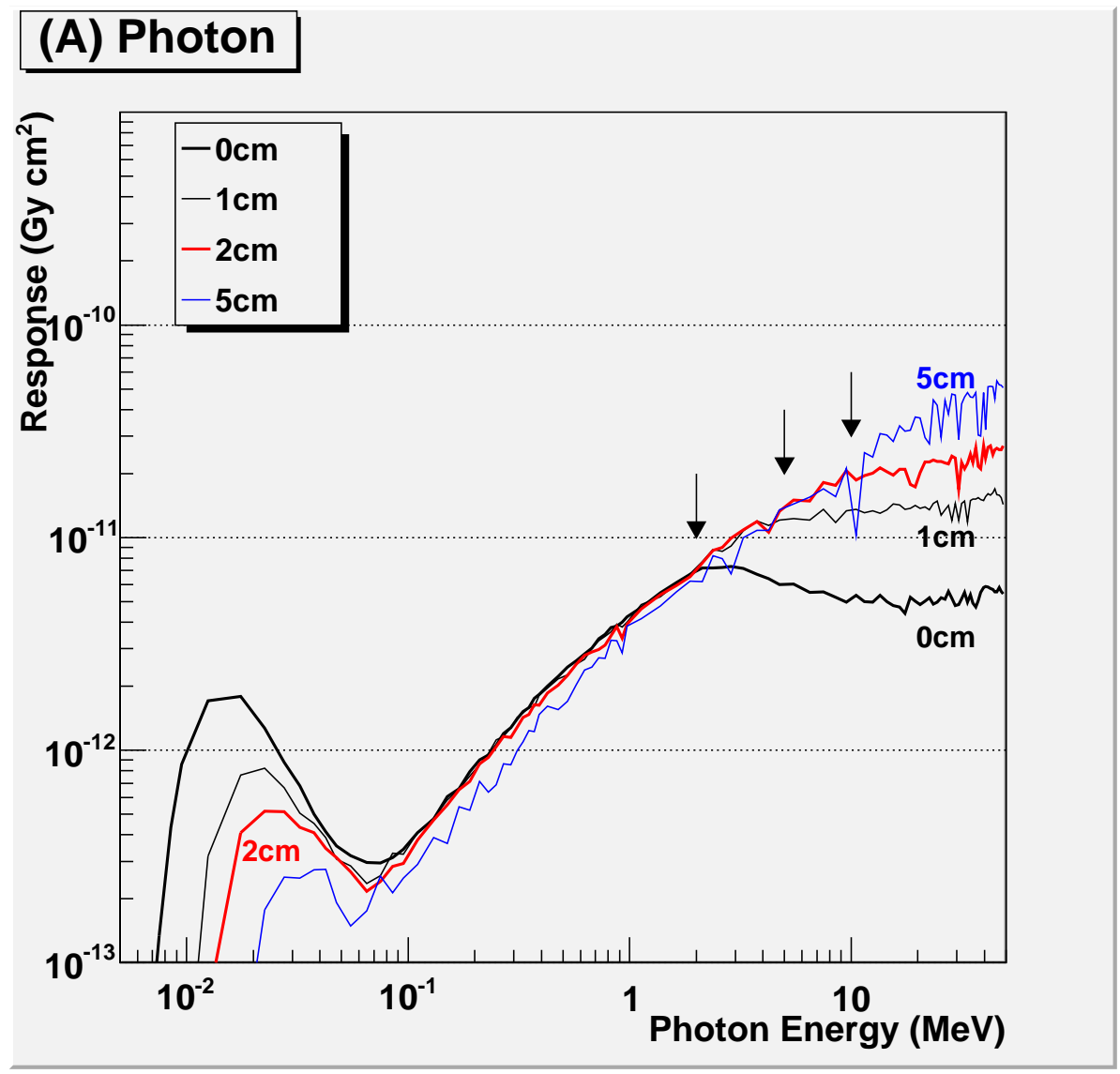

Figure 13. 


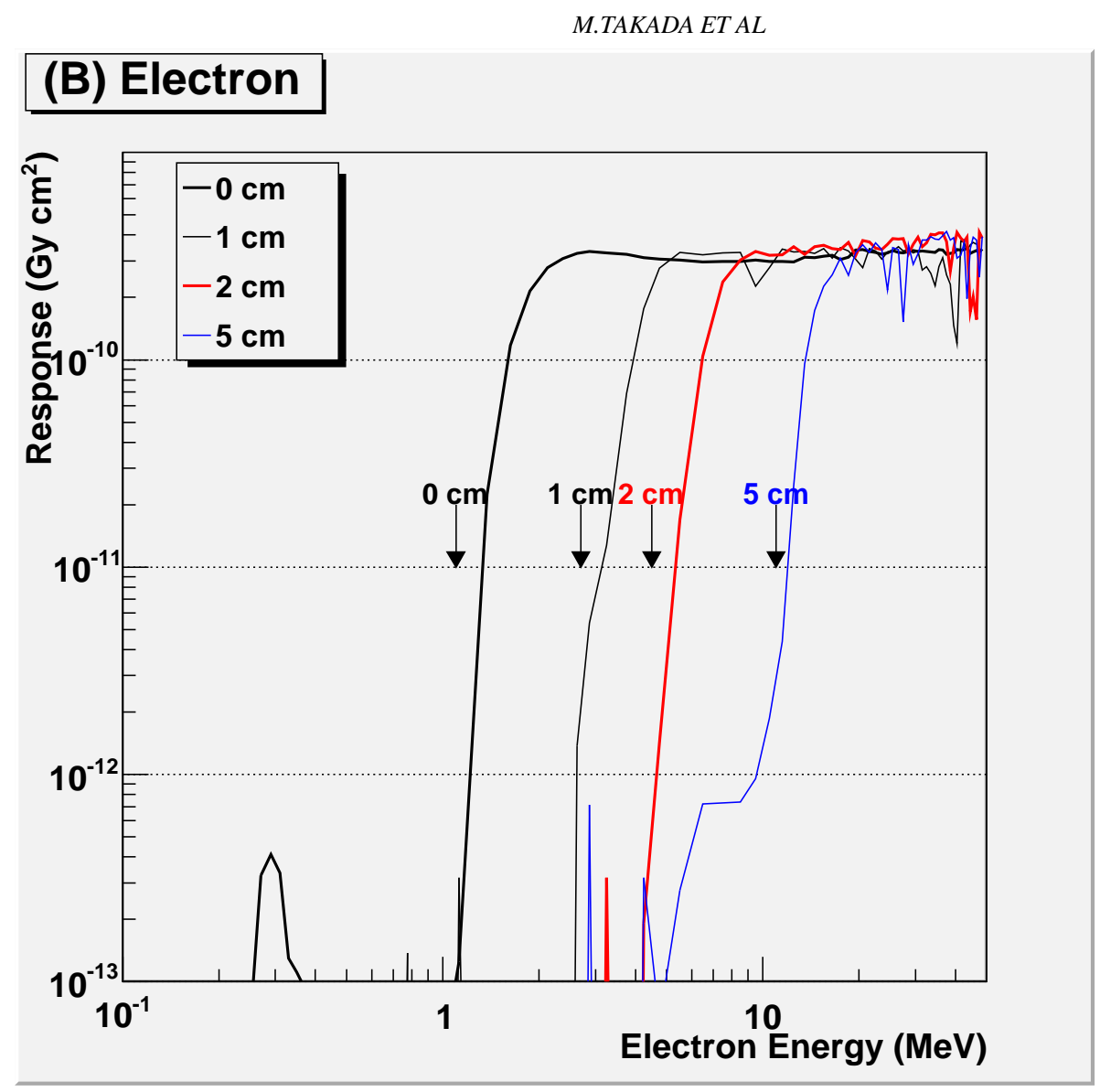

Figure 13. 
ANGLE ABSORBED-DOSE DISTRIBUTION OF BREMSSTRAHLUNG

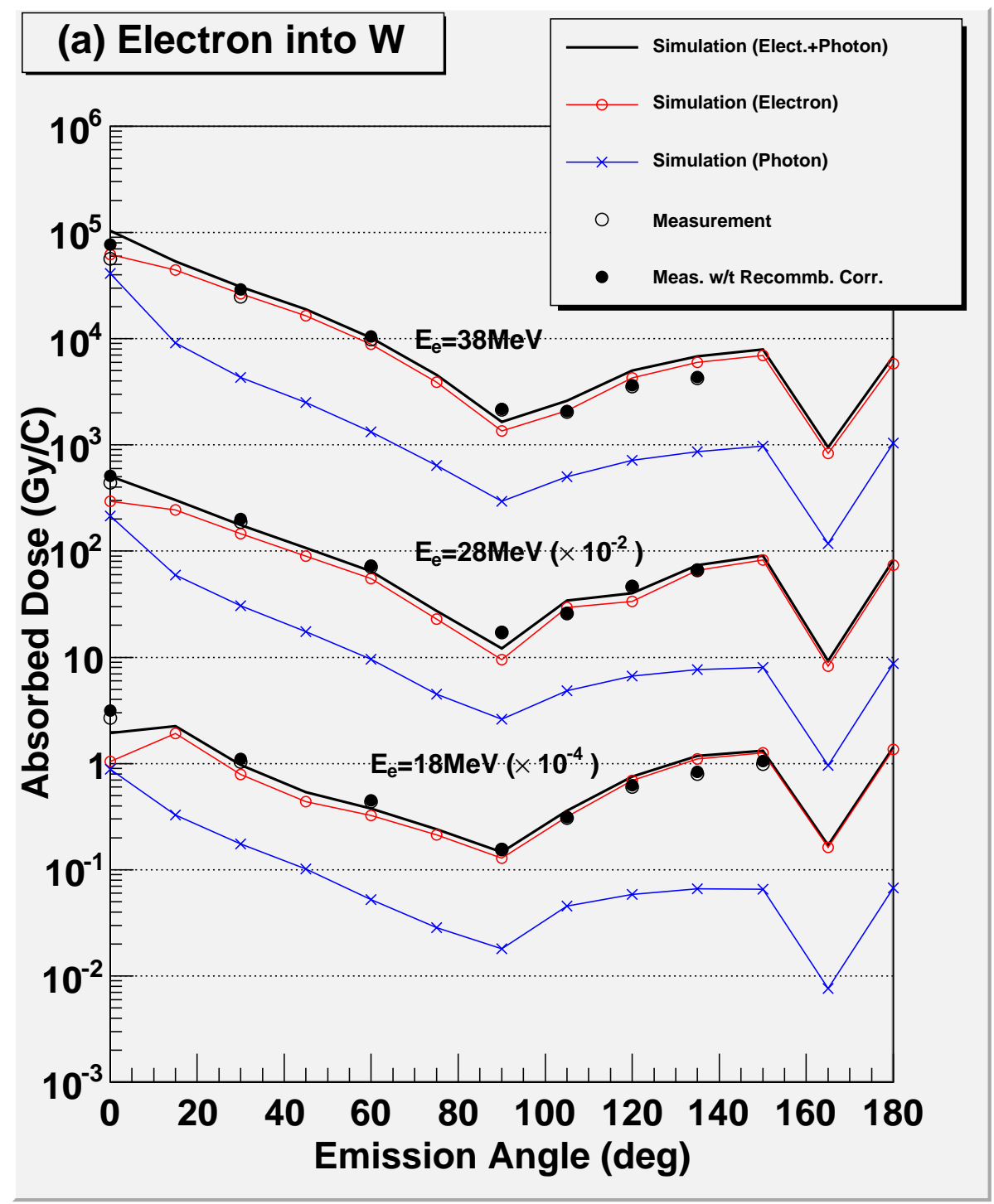

Figure 14. 


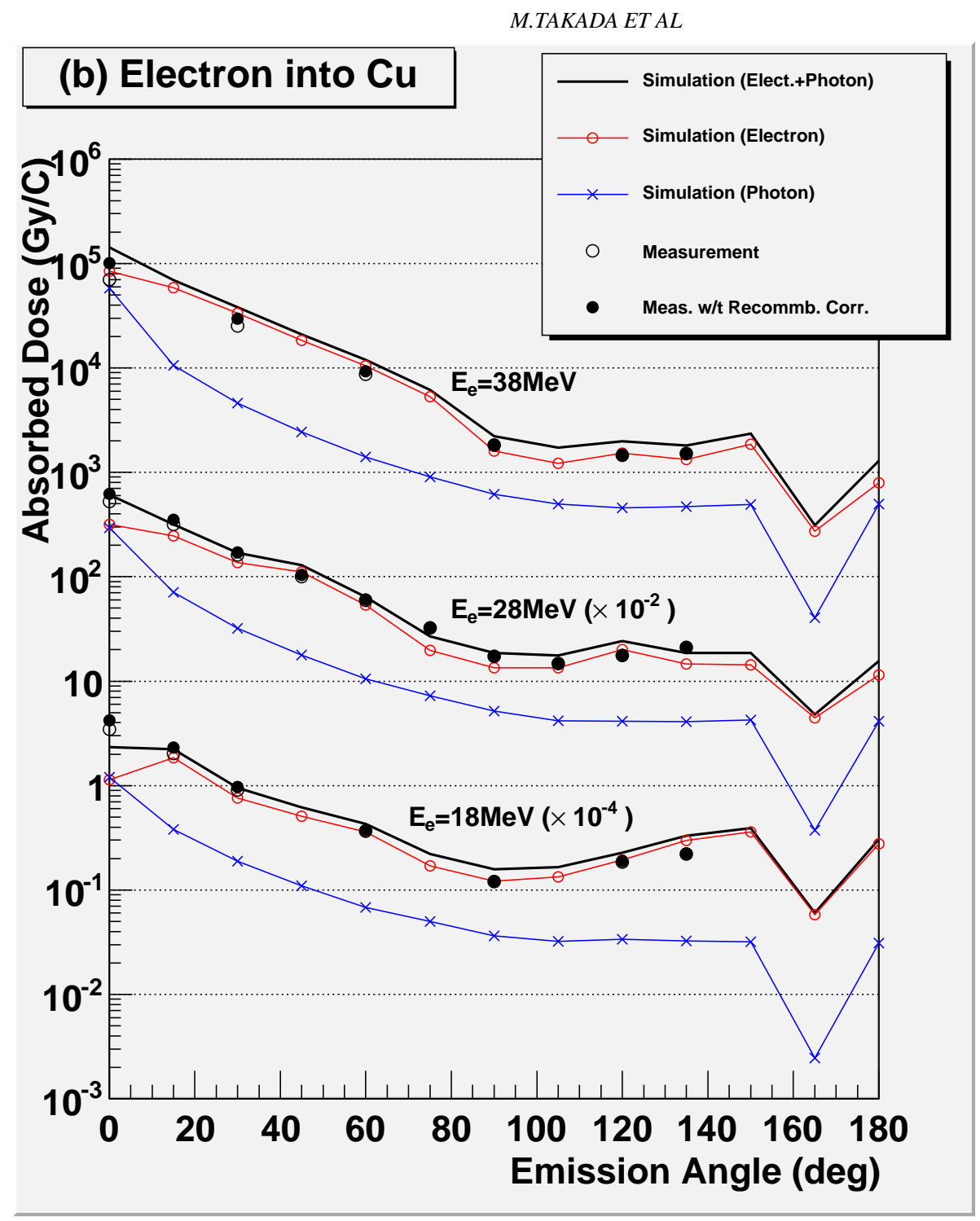

Figure 14. 
ANGLE ABSORBED-DOSE DISTRIBUTION OF BREMSSTRAHLUNG

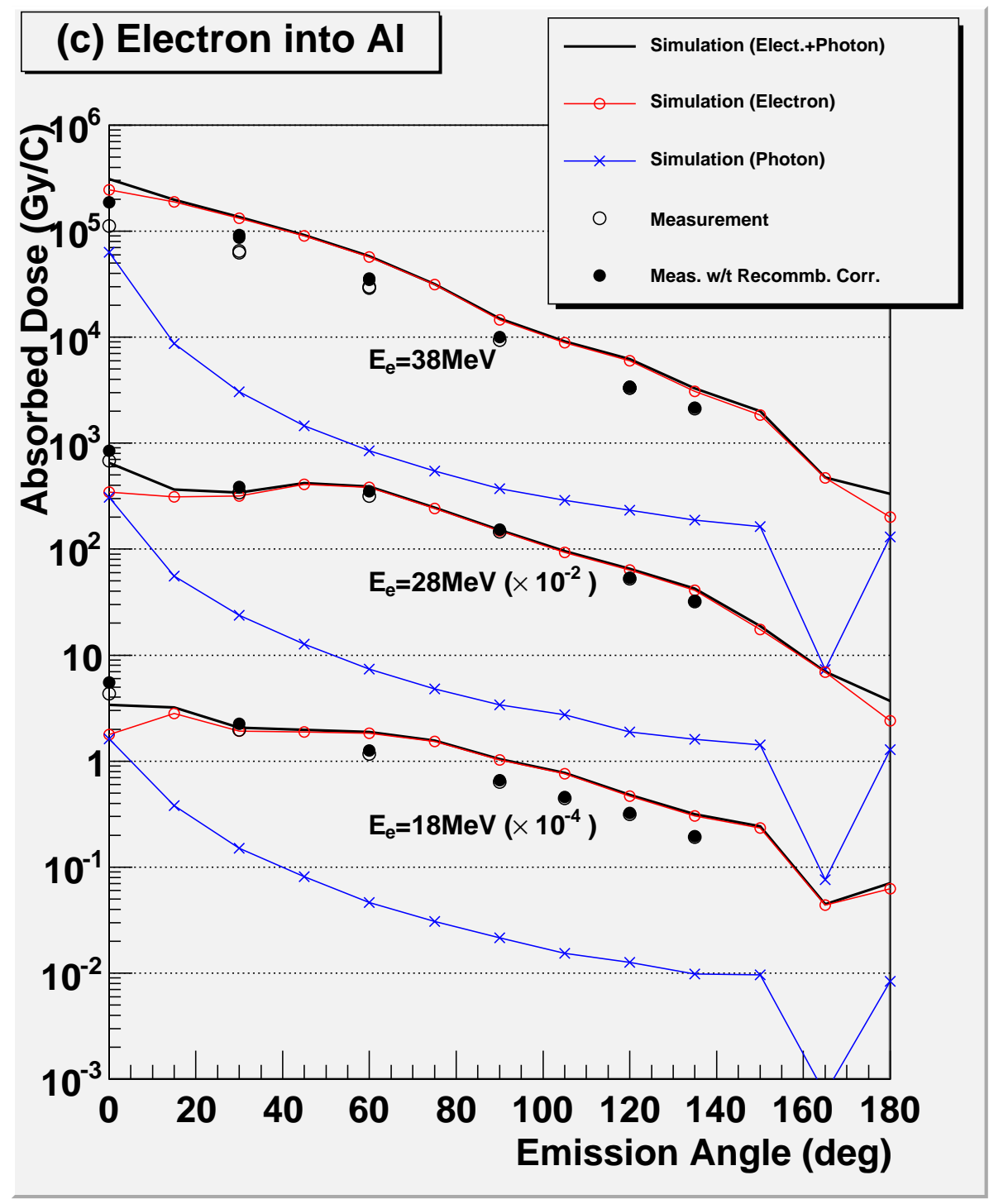

Figure 14. 


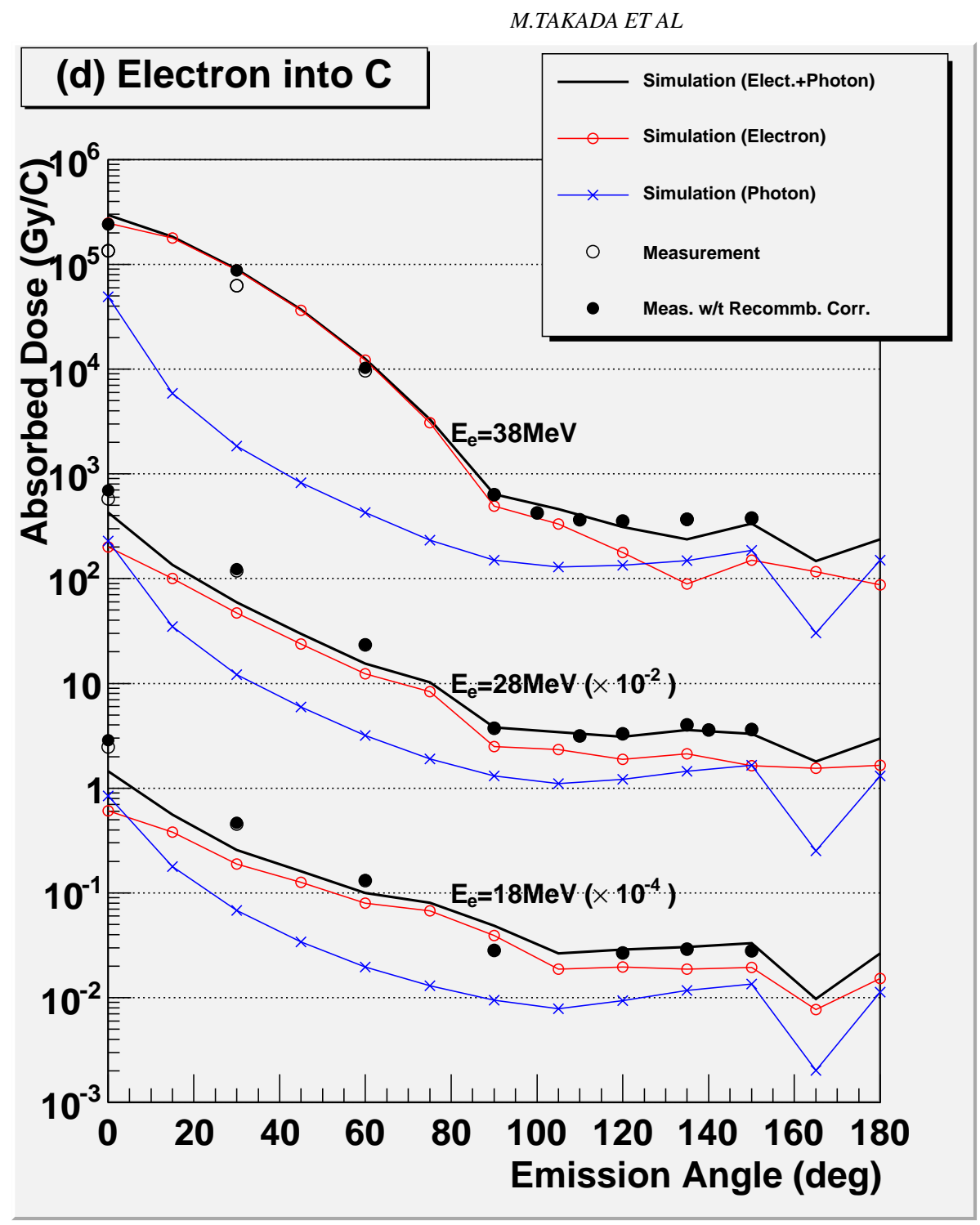

Figure 14. 
ANGLE ABSORBED-DOSE DISTRIBUTION OF BREMSSTRAHLUNG

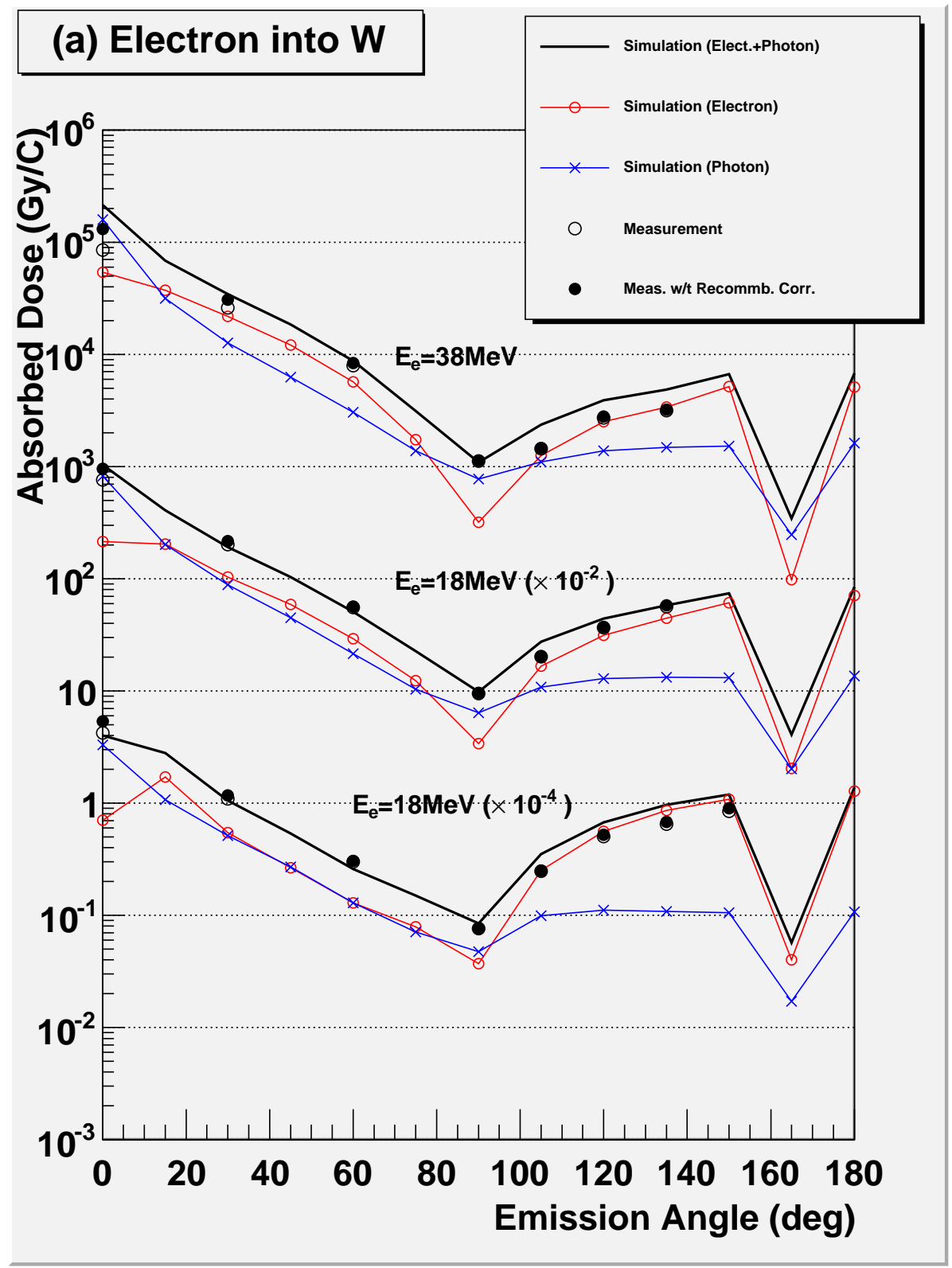

Figure 15 . 


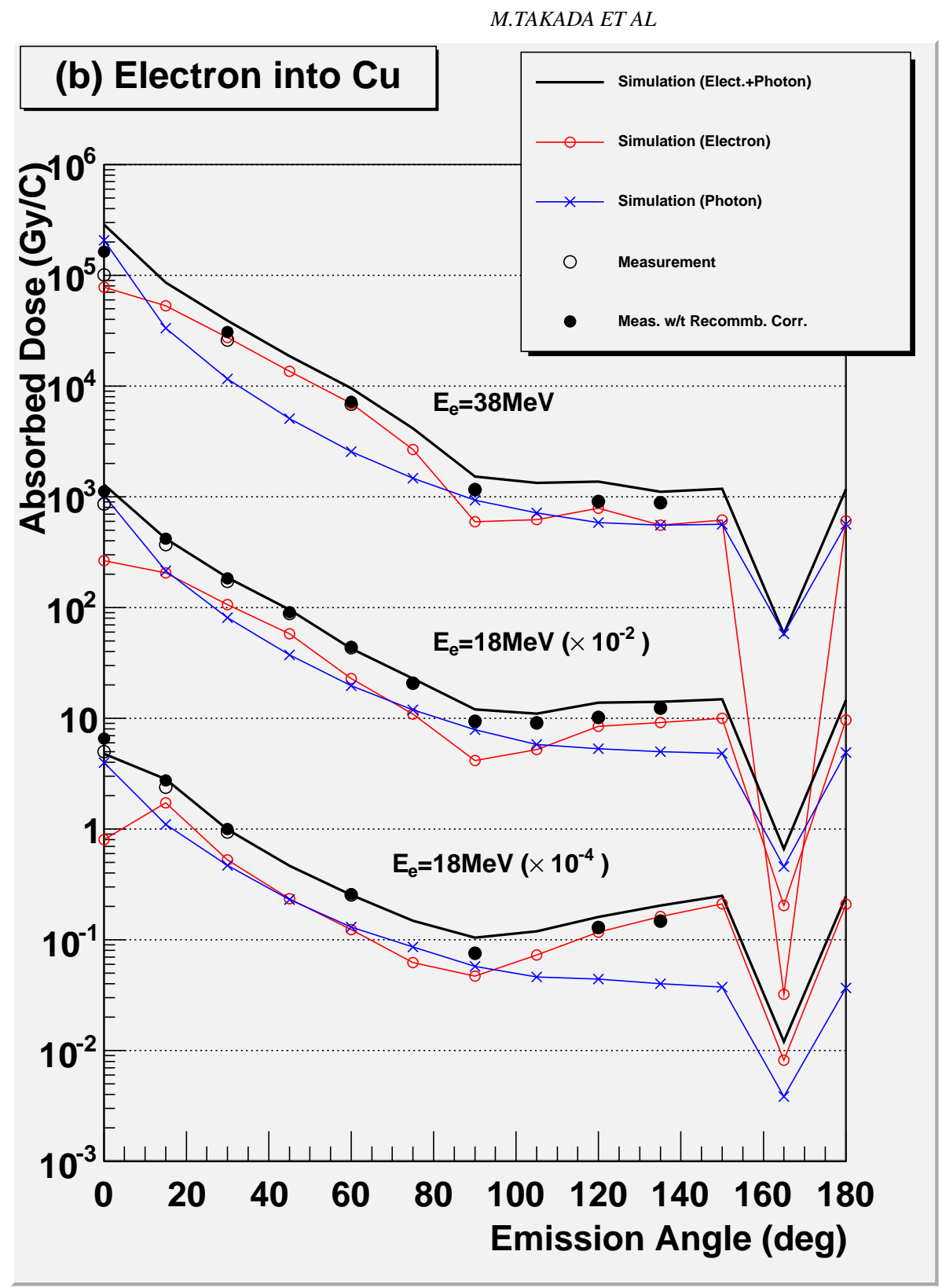

Figure 15 . 
ANGLE ABSORBED-DOSE DISTRIBUTION OF BREMSSTRAHLUNG

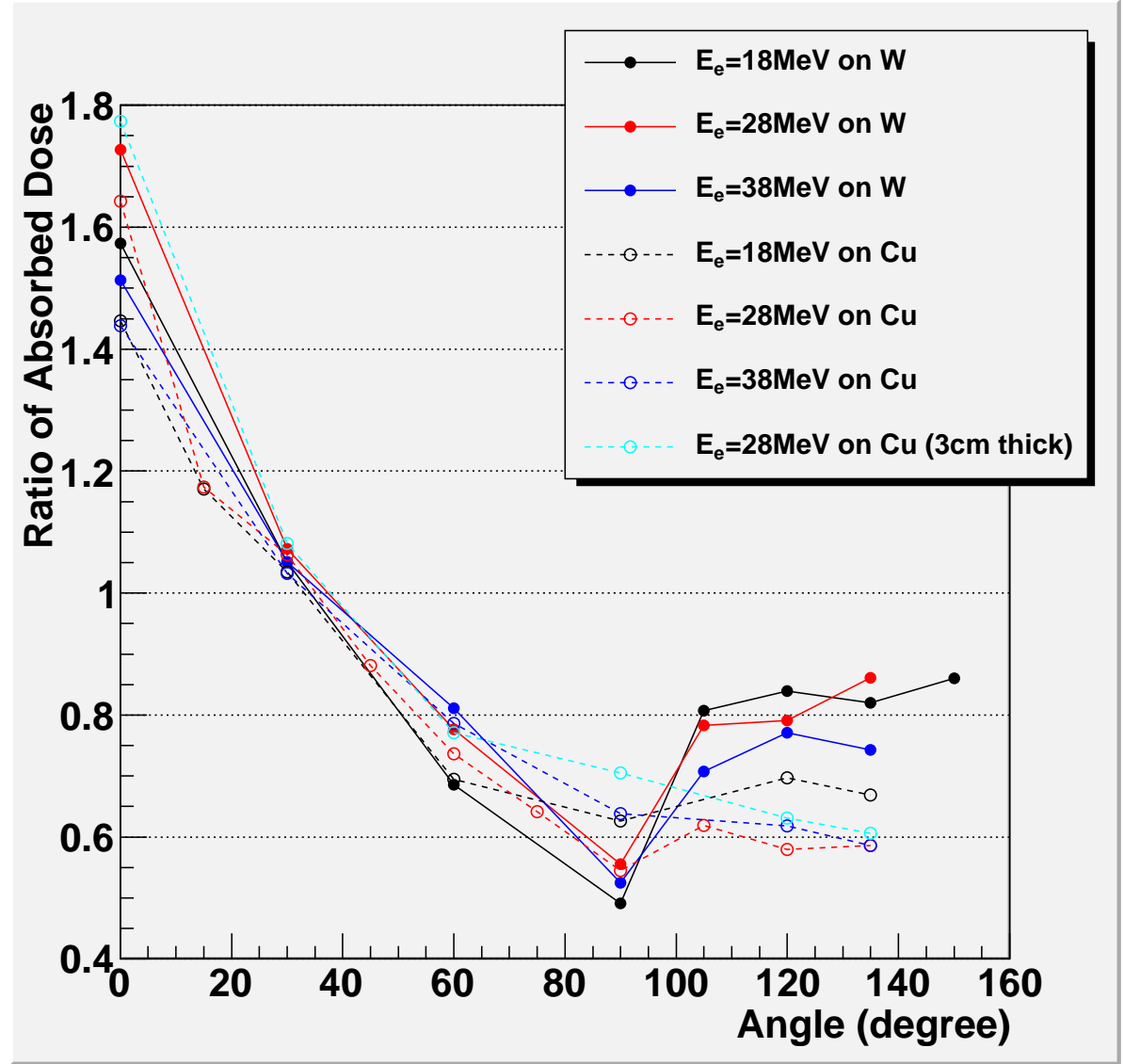

Figure 16. 
M.TAKADA ET AL

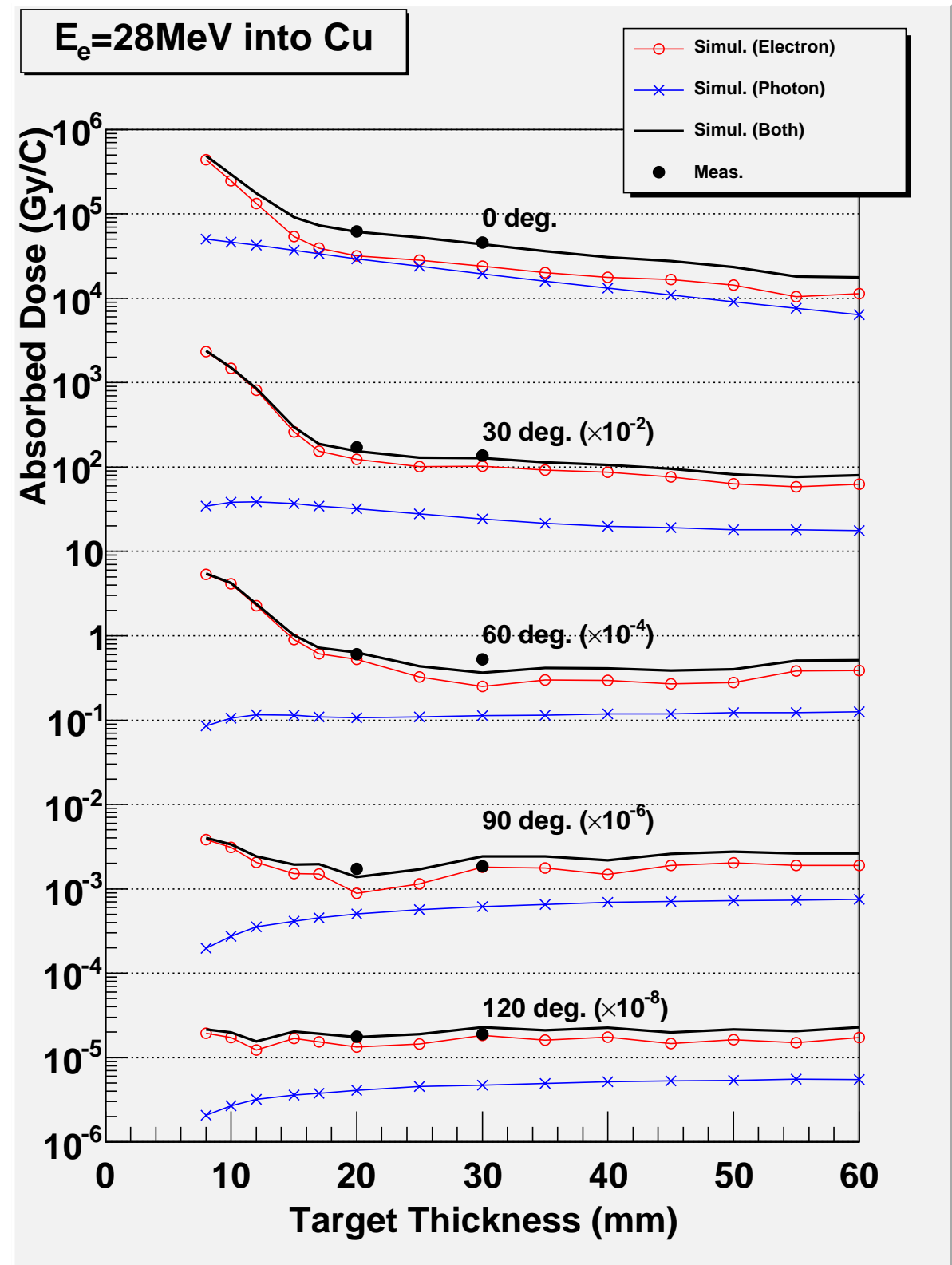

Figure 17. 
ANGLE ABSORBED-DOSE DISTRIBUTION OF BREMSSTRAHLUNG

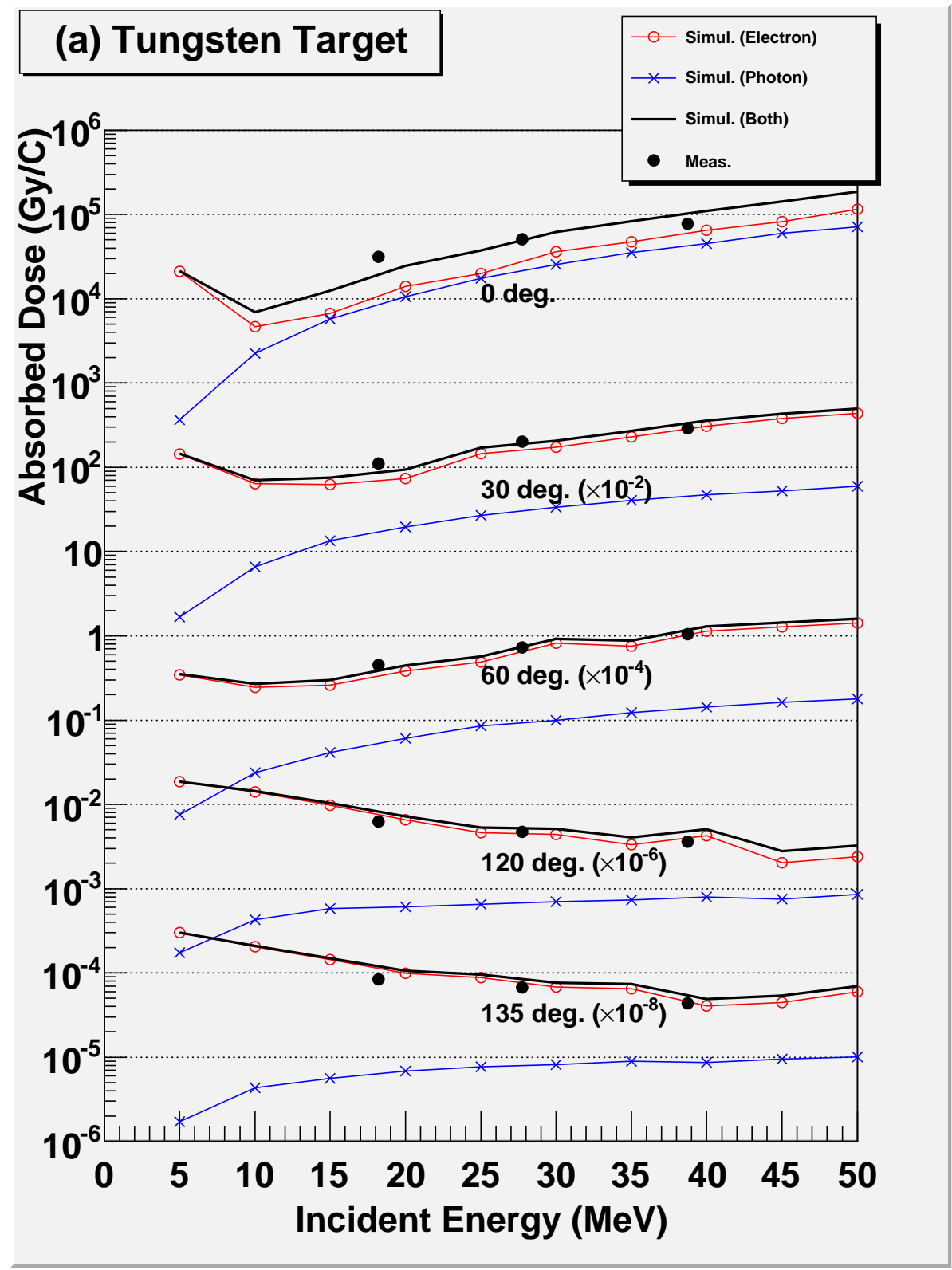

Figure 18. 
M.TAKADA ET AL

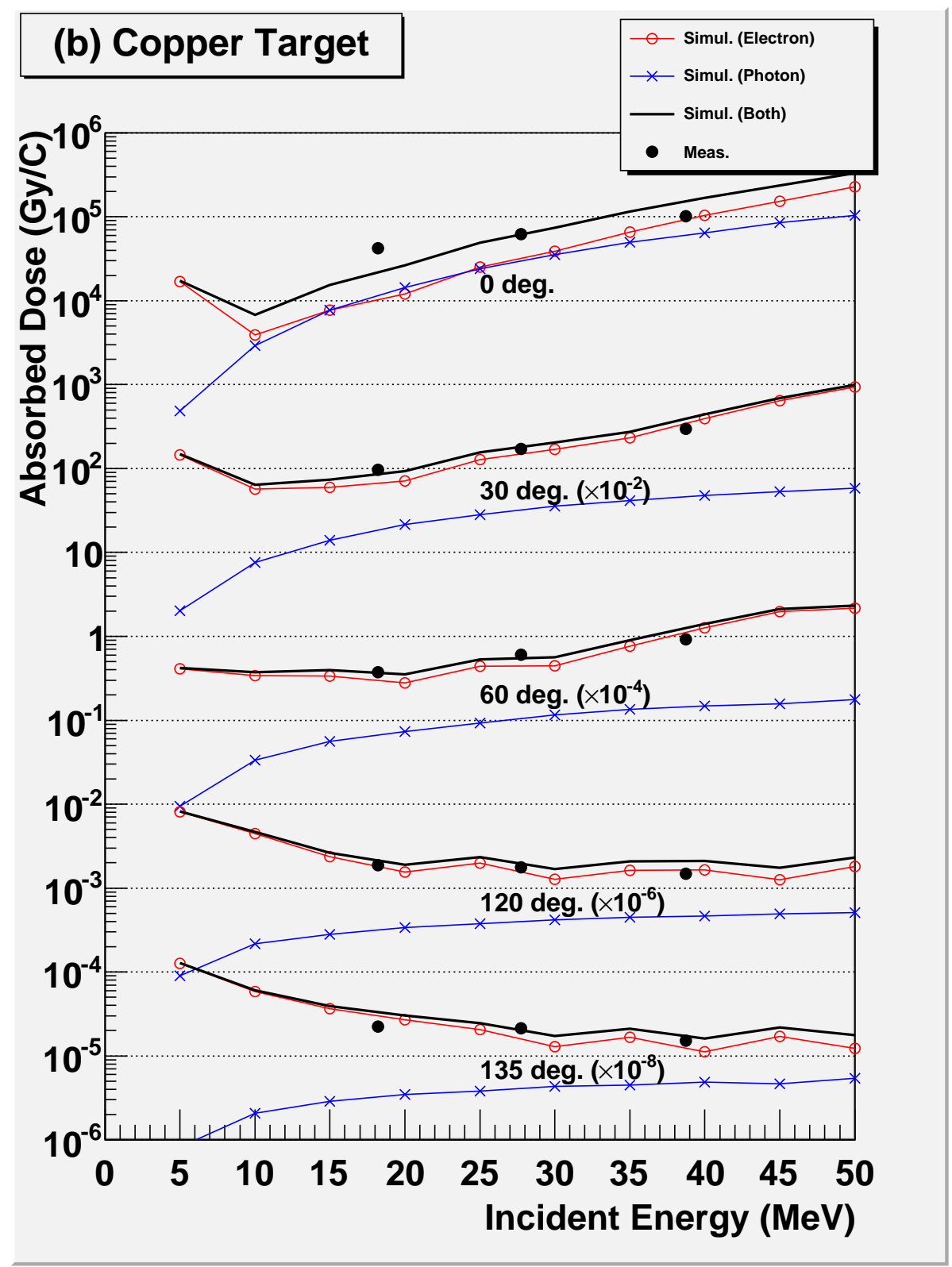

Figure 18. 


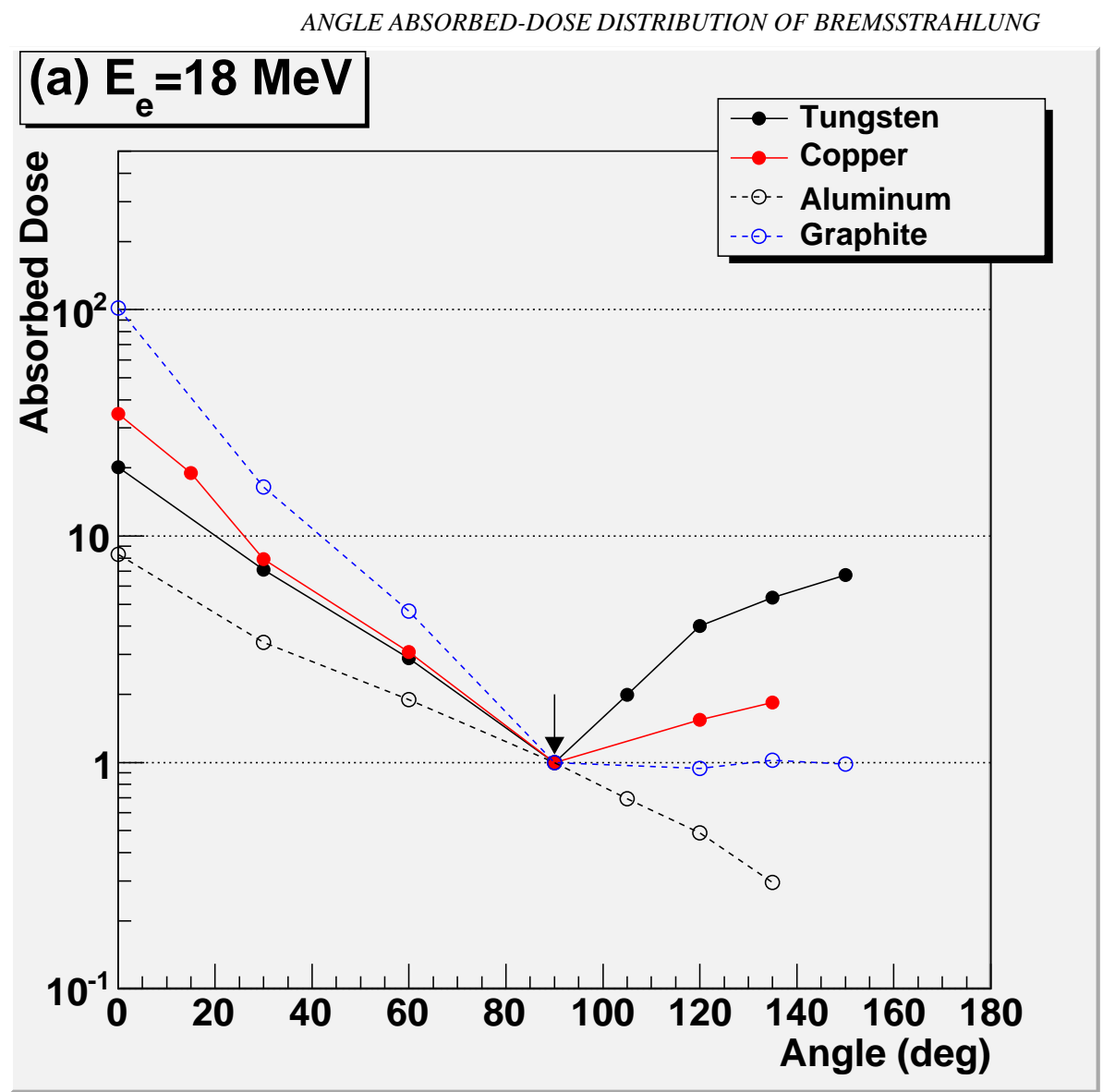

Figure 19. 


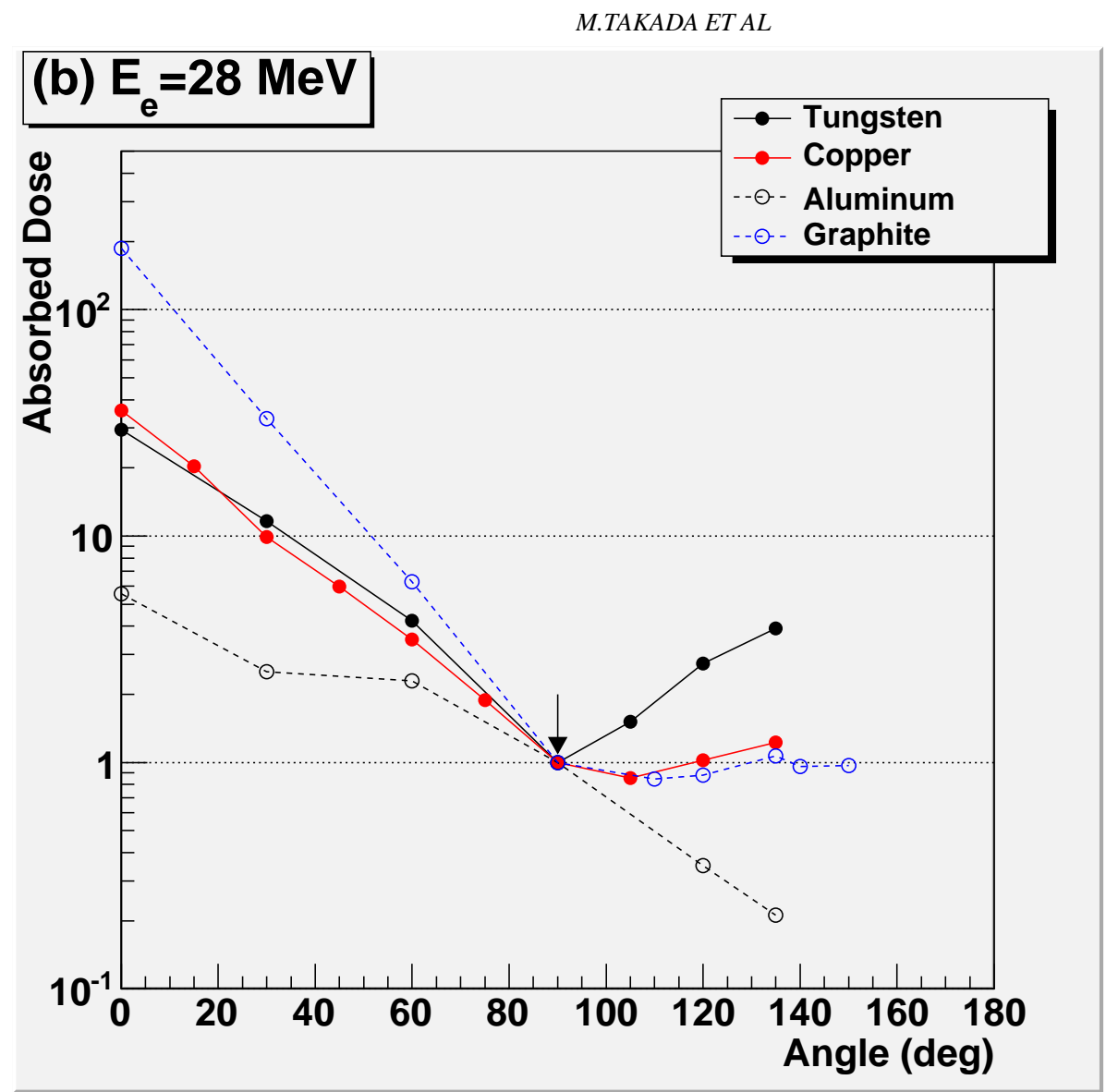

Figure 19. 


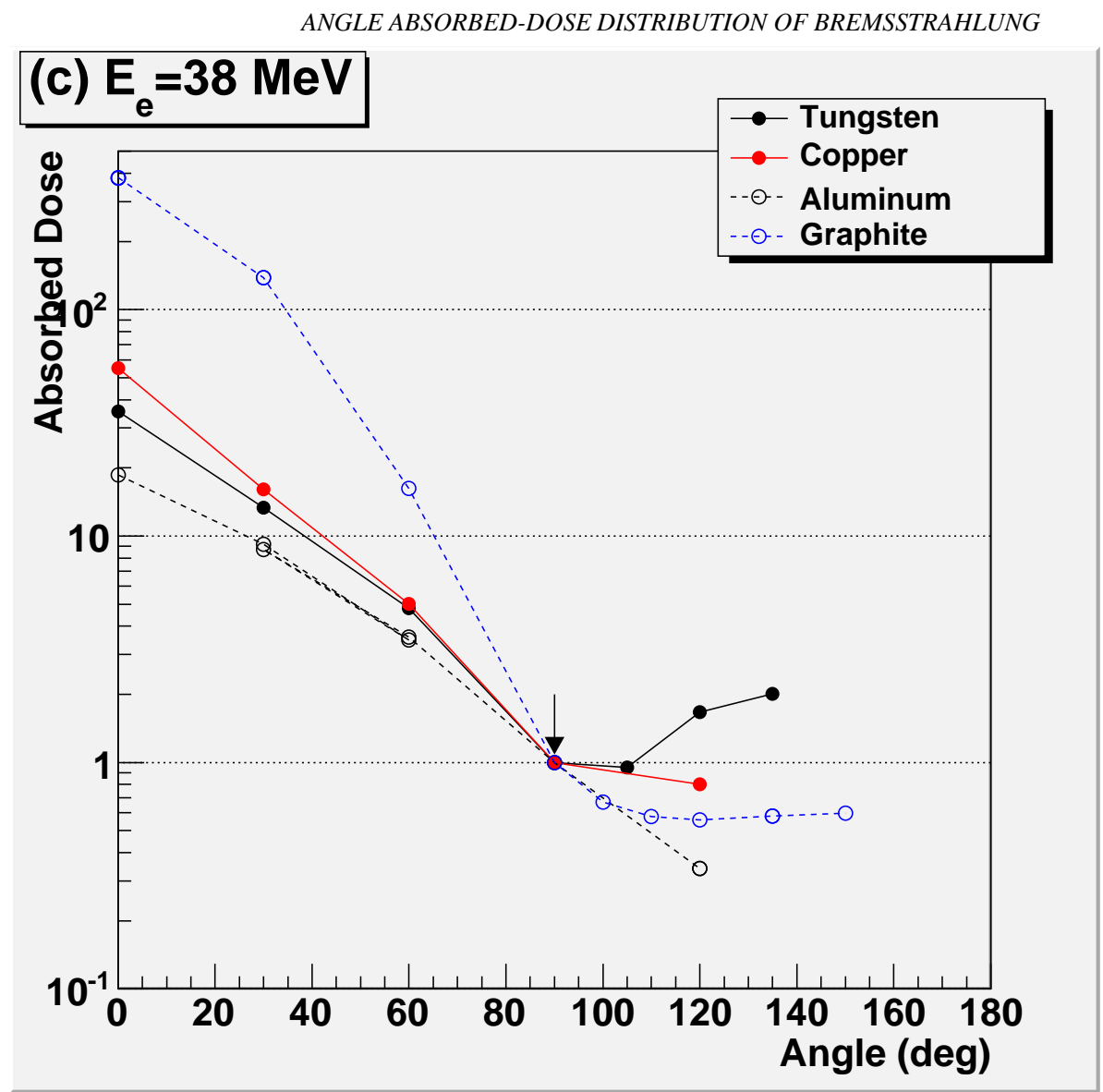

Figure 19. 


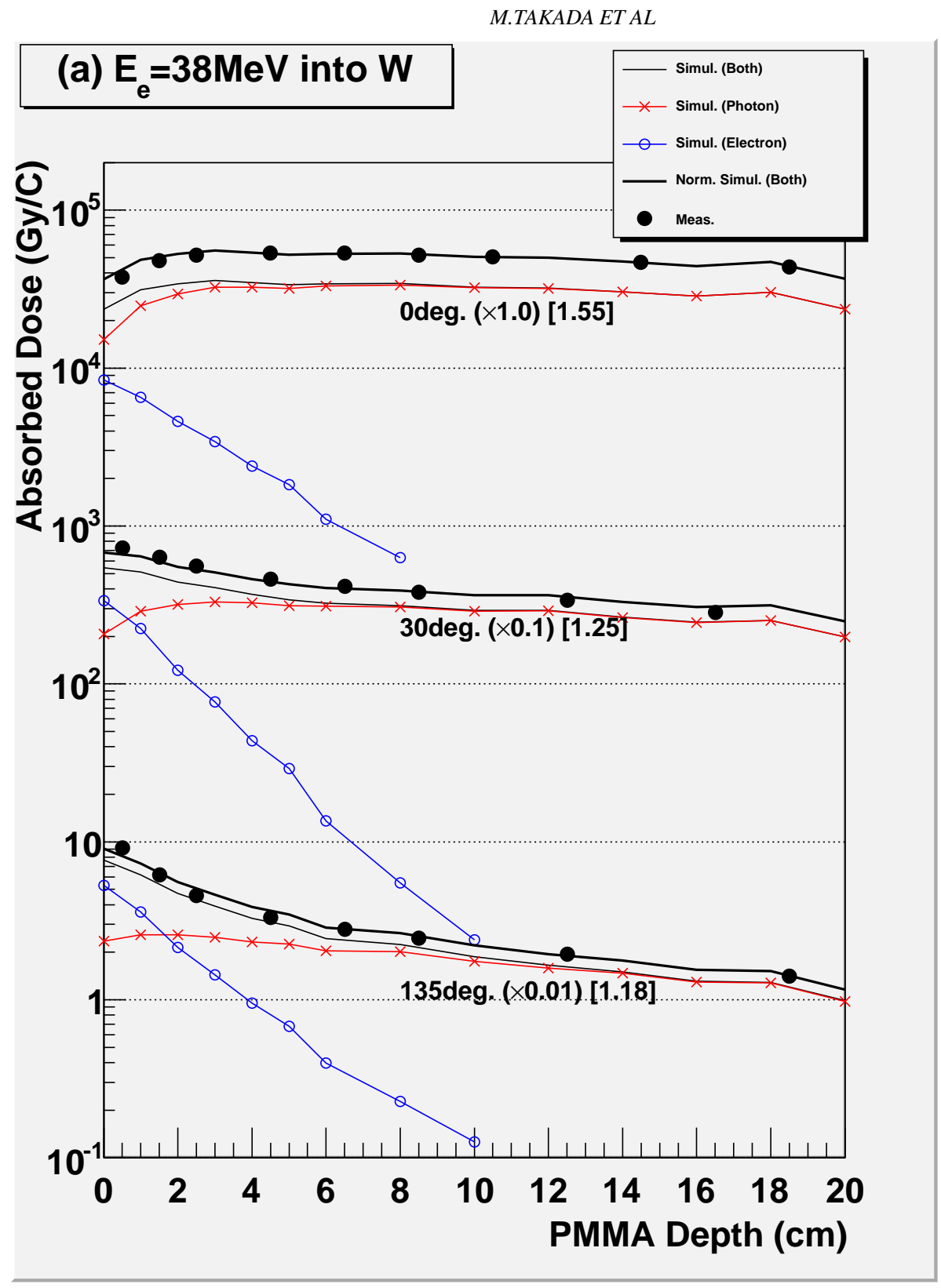

Figure 20 . 
ANGLE ABSORBED-DOSE DISTRIBUTION OF BREMSSTRAHLUNG

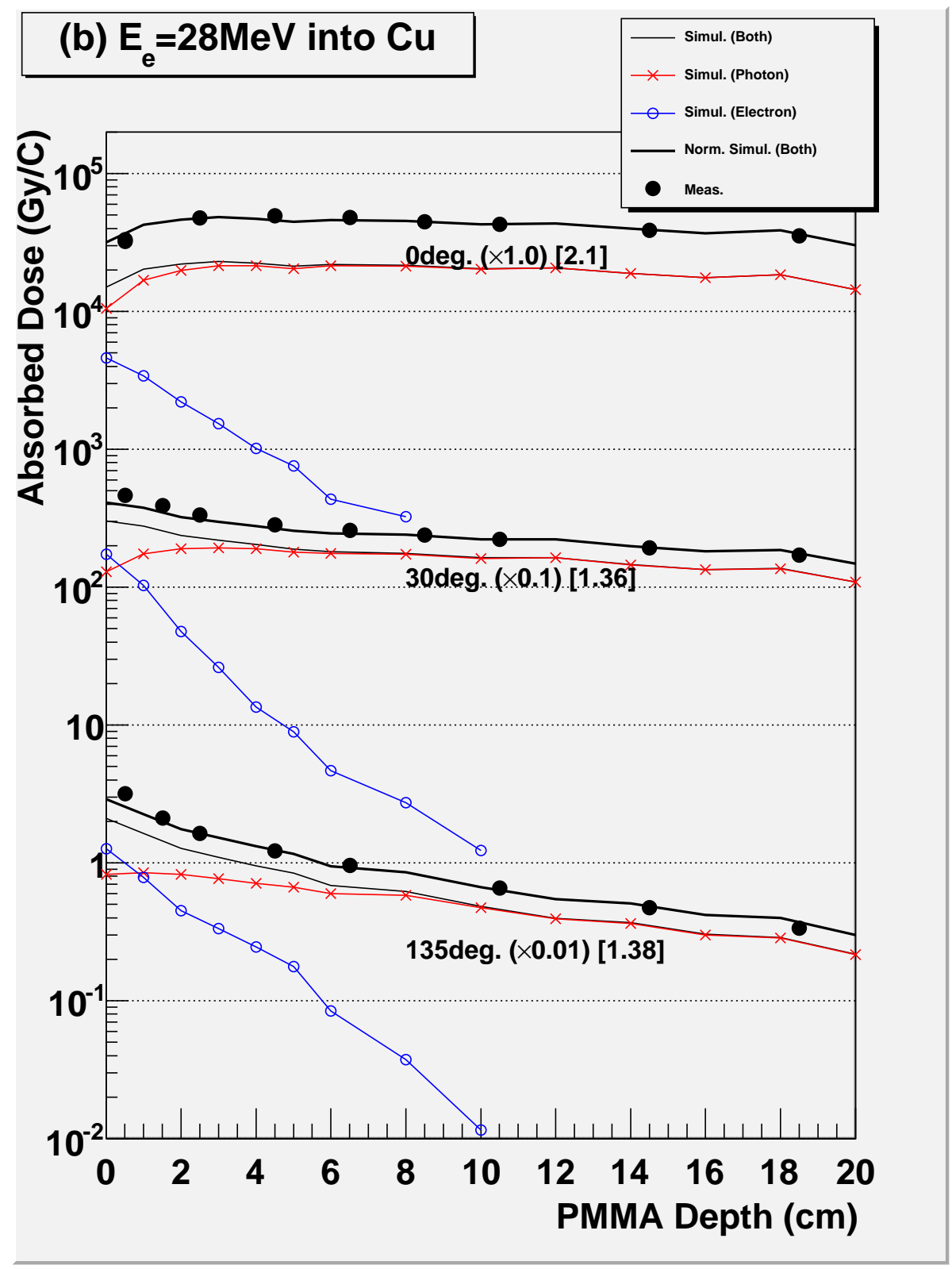

Figure 20. 


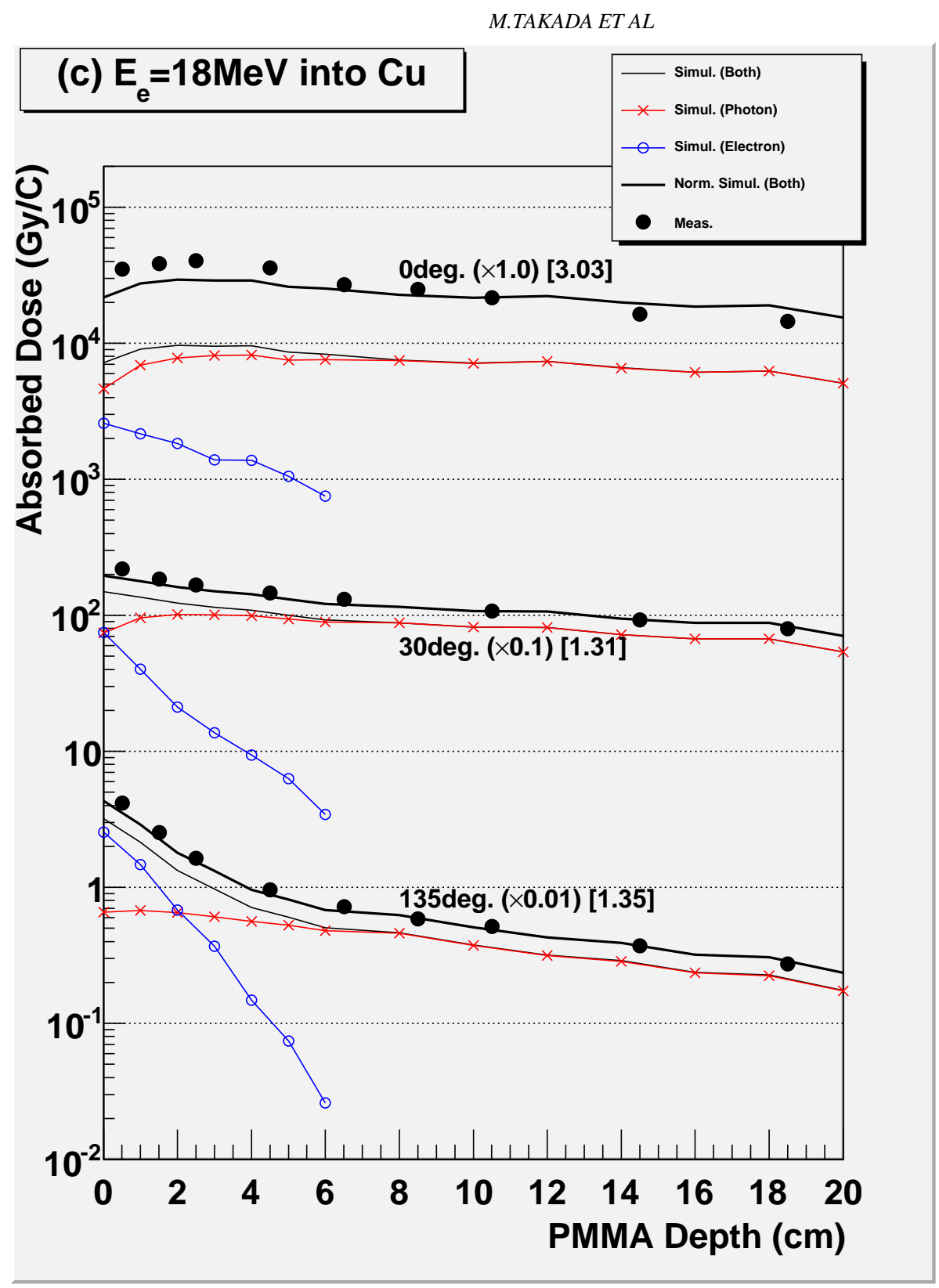

Figure 20 . 